\title{
The Violation of Equivalence Principle and Four Neutrino Oscillations for Long Baseline Neutrinos
}

\author{
Madhurima Pandey ${ }^{a, b} 1$, Debasish Majumdar ${ }^{a}{ }^{2}$, \\ ${ }^{a}$ Theory Division, Saha Institute of Nuclear Physics, HBNI \\ 1/AF Bidhannagar, Kolkata 700064, India \\ ${ }^{b}$ Department of Physics, School of Applied Sciences and Humanities, \\ Haldia Institute of Technology, Haldia, West Bengal, 721657, India. \\ Amit Dutta Banik ${ }^{c} 3$ \\ ${ }^{c}$ Key Laboratory of Quark and Lepton Physics (MoE) and Institute of Particle \\ Physics, Central China Normal University, Wuhan 430079, China \\ Ashadul Halder ${ }^{d} 4$ \\ ${ }^{d}$ Department of Physics, St. Xavier's College, \\ 30, Mother Teresa Sarani, Kolkata - 700016, India
}

\begin{abstract}
Violation of equivalence principle predicts that neutrinos of different flavour couple differently with gravity. Such a scenario can give rise to gravity induced flavour oscillations in addition to the usual mass flavour neutrino oscillations during the neutrino propagation. Even if the equivalence principle is indeed violated, their measure will be extremely small. We explore the possibility to probe the violation of equivalence principle (VEP) for the case of long baseline (LBL) neutrinos in a 4-flavour neutrino framework (3 active +1 sterile) where both mass and gravity induced oscillations are considered. To this end, we have explicitly calculated the oscillation probability in 4-flavour framework that includes in addition to the mass-flavour mixing in matter, the gravity-flavour mixing also. The energy eigenvalues are then obtained by diagonalising such a 4-flavour mixing matrix. The formalism is then employed to estimate the wrong and right sign muon yields at a far detector for neutrinos produced in a neutrino factory and travel through the Earth matter. These results are compared with the similar estimations when the ususal three active neutrinos are considered.
\end{abstract}

\footnotetext{
${ }^{1}$ email: madhurima0810@gmail.com

${ }^{2}$ email: debasish.majumdar@saha.ac.in

3 email: amitdbanik@mail.ccnu.edu.cn

${ }^{4}$ email: ashadul.halder@gmail.com
} 


\section{Introduction}

The oscillatory nature of neutrinos [1] from one type of flavour to another is now well established by various terrestrial experiments with neutrinos having natural origin such as atmospheric, solar and man made such as reactor $[2,3,4]$ or accelerator neutrinos. The mass eigenstates and the weak interaction eigenstates of neutrinos not being the same, neutrino flavour eigenstate in a coherent neutrino beam can oscillate into a eigenstate having different flavour after traversing a distance. These oscillations occur due to the phase difference that is acquired by a neutrino eigenstate during its propagation and this phase difference depends on the baseline length and the mass square difference of two neutrino mass eigenstates. The massive nature of neutrinos is established by experimental phenomenon of the oscillations. The framework of Standard Model (SM) of particle physics does not have mechanisms to explain how the neutrinos acquire masses and theories beyond the SM framework needs to be invoked for explaining the neutrino mass.

In association to the neutrino mass, violation of the equivalence principle (VEP) $[5,6]$ can also induce neutrino oscillations. If the equivalence principle is indeed violated in nature, different types of neutrinos couple differently with gravity which means that different neutrino flavour eigenstates interact with the gravitation field with different strengths. Thus in this situation the gravitational coupling (constant) $G$ is different for different types of neutrinos. This leads to the fact that the gravity eigenstates of neutrinos are not identical as those of their weak interaction eigenstates. An important feature of Einstein's general theory of relativity is the equivalence principle which affirms that the inertial mass and the gravitational mass are the same. This is stated that an observer standing on the Earth experiences the gravitational force which is same as the pseudo force experienced by the same observer in accelerated reference frame. Therefore, if the equivalence principle is indeed violated, then the coupling strengths of neutrinos with the gravitational field as well as the gravitational constant $(G)$ are no more universal.

A general consequence of VEP is the gravitational redshift - while propagating through a gravitational field the energy $E$ of a neutrino will be shifted by an amount $E^{\prime}=\sqrt{g_{00}} E=$ $E\left(1-\frac{G M}{R}\right)=E(1+\phi)[6,7]$, where the gravitational potential $(\phi)[8]$ is defined as $\phi=\frac{G M}{R}$, $M$ and $R$ being the mass of the source and the distance over which the gravitational field operates respectively ${ }^{5}$. By virtue of the equivalence principle, energies of different types of neutrinos are shifted by an equal amount and eventually while they traverse through the

\footnotetext{
${ }^{5}$ In the presence of gravitational field, the proper time in a curve manifold is $d \tau=\sqrt{g_{\mu \nu} d x^{\mu} d x^{\nu}}$ which can lead to the relation $E^{\prime}=\sqrt{g_{00}} E$. The proper time $(d \tau)$ relates to the coordinate time ( $d t$ ) through $d \tau=\sqrt{g_{\mu \nu} d x^{\mu} d x^{\nu}}$ (when clock is at rest). If a distant star is emitting $N$ number of waves having frequency $f_{\text {star }}$ and proper time interval $\Delta \tau_{\text {star }}$ respectively and if the Earth is detecting the same with frequency $f_{\text {Earth }}$ and proper time interval $\Delta \tau_{\text {Earth }}$, then $\frac{f_{\text {star }}}{f_{\text {Earth }}}=\frac{\Delta \tau_{\text {Earth }}}{\Delta \tau_{\text {star }}}=$
} 
gravitational field, the phase difference between two types of neutrinos may not be generated in this case. But if the violation of equivalence principle is violated then for different types of neutrinos the energies will be shifted differently. The VEP will induce a phase $\sim \Delta E L$, $\Delta E=\left|E_{i}-E_{j}\right|, E_{i}$ and $E_{j}$ being the red-shifted energies of the neutrino species $i$ and $j$ respectively and $L$ defines the baseline length from the source to the Earth through which neutrino propagates. $E_{i}, E_{j}$ are the energy eigenstates in gravity basis. Similar to the case for mass-flavour oscillations, the acquired phase difference will generate a gravity induced oscillations between different flavours of neutrinos having the oscillatory part $\sim|\Delta E L|=$ $\left|\Delta f_{i j}\right| L E$, where $\left|\Delta f_{i j}\right|=\left|f_{i}-f_{j}\right|, f_{i}$ is defined as $f_{i}=\frac{G_{i} M}{R}=\left(\frac{G M}{R}\right) \alpha_{i}=\phi \alpha_{i}, G_{i}$ being the gravitational coupling of the neutrino having index $i$.

In this work, we study the effects of violation of equivalence principle with three active and one sterile neutrino ${ }^{6}$. To this end, we first obtain the evolution equation of the neutrino including three effects namely the mass-flavour mixing, the gravity-flavour mixing and the matter effect. The $(4 \times 4)$ evolution matrix is then diagonalised by proper unitary transformation and the energy eigenvalues are obtained explicitly. This enables to compute the phase difference between any two neutrinos during its propagation and hence the expressions for different oscillation probabilities with the oscillatory part have been written. Using the latest experimental limits on active-sterile neutrino mixing and mass square difference $\Delta m_{41}^{2}$ for normal hierarchy of neutrino mass eigenstates (and assumed normal hierarchy for neutrino gravity eigenstates) and best fit values of active neutrino mixing parameters (mixing angles and mass square differences), we obtain new four flavour gravity induced neutrino oscillation probabilities which also include the matter effect induced by the matter through which the neutrinos travel. We show that VEP will induce new set of parameters $\Delta f_{i j}$ which change the neutrino oscillation probabilities significantly. We demonstrate this in case of a neutrino beam propagating through a baseline of $7000 \mathrm{~km}$ inside Earth matter. Therefore, even if VEP is very small, it will significantly affect the number of muon yields (from $\nu_{\mu}$ ) at the far detector in a long baseline (LBL) neutrino experiment. In this work, we compute our results of these neutrino yields considering an LBL neutrino experiment for a baseline length of around $7000 \mathrm{~km}$ with the end detector to be a iron calorimeter (ICAL) of $50 \mathrm{kTon}$ such as the one proposed for the India-based Neutrino Observatory or INO [10] and at the origin of the neutrino source is from a neutrino factory or accelerator such as CERN. We calculate the number of right sign and wrong sign (explained later) muon yields at the end detector and their variations with the change in $\Delta f_{i j}$ (the VEP effect). We also mention

\footnotetext{
$\frac{\sqrt{g_{00}\left(x_{\text {Earth }}\right)}}{\sqrt{g_{00}\left(x_{\text {star }}\right)}}=\sqrt{\left(\frac{1+2 \phi_{\text {Earth }}}{1+2 \phi_{\text {star }}}\right)}=1+|\Delta \phi|$.

${ }^{6} \mathrm{In}$ a previous work, velocity induced oscillations in matter had been addressed [9] in case of long baseline neutrinos similar to the present analysis. But this work was performed in the context of only three active neutrinos.
} 
that perhaps this is for the first time, neutrino oscillations in 4-flavour scenario has been worked out in detail and expressions for oscillation probabilities are obtained incorporating both gravity induced and matter induced mass flavour oscillations.

We organise the paper in the following manner. In Section 2 we present a brief discussion about the formalism of gravity induced as well as mass induced oscillations in matter within a 4-flavour framework. The calculational results are furnished in Section 3 which is divided into two subsections. In Section 3.1 we discuss about how the oscillation probabilities vary with the gravity effects, while Section 3.2 deals with the possible neutrino induced muon yield in long baseline (LBL) experiments in the presence of both gravity induced oscillations and mass induced oscillations in matter. Finally in Section 4 the paper is summarized with some discussions.

\section{Neutrino Oscillations in Matter with VEP in 4- flavour Scenario}

Neutrino oscillations would arise because of the non zero nature of neutrino masses. The essence of this phenomena was first observed by Pontecorvo [11, 12] in 1957, while Maki, Nakagawa and Sakata (1962) first pointed out the possibility of the arbitrary mixing between the two massive neutrino states.

In the case of massive neutrinos, the neutrino flavour eigenstates $\left|\nu_{\alpha}\right\rangle$ produced in a charged current weak interactions can be expressed as the linear combination of the mass eigenstates $\left|\nu_{i}\right\rangle$ via a unitary mixing matrix $U$ (with matrix elements denoted as $U_{\alpha i}^{*}$ ).

$$
\left|\nu_{\alpha}\right\rangle=\sum_{i=1}^{n} U_{\alpha i}^{*}\left|\nu_{i}\right\rangle,
$$

where the number of neutrino species is indicated by $n$. In what follows we consider the mixing matrix $U$ to the real (no $\mathrm{CP}$ violating phases) and for a 4-flavour scenario $U \equiv U_{(4 \times 4)}$. In this work we consider an extra sterile neutrino $\left(\nu_{s}\right)$ in addition to the three active neutrinos $\left(\nu_{e}, \nu_{\mu}, \nu_{\tau}\right)$. For this 4 -flavour $(3$ active +1 sterile) scenario, the relation between the neutrino flavour eigenstates and the mass eigenstates can be parameterized as

$$
\left(\begin{array}{c}
\nu_{e} \\
\nu_{\mu} \\
\nu_{\tau} \\
\nu_{s}
\end{array}\right)=U_{(4 \times 4)}\left(\begin{array}{c}
\nu_{1} \\
\nu_{1} \\
\nu_{3} \\
\nu_{4}
\end{array}\right)=\left(\begin{array}{cccc}
U_{e 1} & U_{e 2} & U_{e 3} & U_{e 4} \\
U_{\mu 1} & U_{\mu 2} & U_{\mu 3} & U_{\mu 4} \\
U_{\tau 1} & U_{\tau 2} & U_{\tau 3} & U_{\tau 4} \\
U_{s 1} & U_{s 2} & U_{s 3} & U_{s 4}
\end{array}\right)\left(\begin{array}{c}
\nu_{1} \\
\nu_{1} \\
\nu_{3} \\
\nu_{4}
\end{array}\right),
$$

where $U_{\alpha i}$ etc. are the elements of the Pontecorvo - Maki - Nakagawa - Sakata (PMNS) matrix $U_{(4 \times 4)}[13]$. 
The PMNS matrix $U_{(4 \times 4)}$ depends on the mixing angles and CP violating phases. In this work, we assume CP conservation in the neutrino sector. The PMNS matrix can now be described in terms of the successive rotations $(R)$, which are functions of the six mixing angles $\theta_{14}, \theta_{24}, \theta_{34}, \theta_{13}, \theta_{12}, \theta_{23}$ in 4 -flavour scenario $[14,15]$.

$$
U_{(4 \times 4)}=R_{34}\left(\theta_{34}\right) R_{24}\left(\theta_{24}\right) R_{14}\left(\theta_{14}\right) R_{23}\left(\theta_{23}\right) R_{13}\left(\theta_{13}\right) R_{12}\left(\theta_{12}\right),
$$

where these six orthogonal matrices $(R)$ can be written as

$$
\begin{aligned}
R_{34}\left(\theta_{34}\right)= & \left(\begin{array}{cccc}
1 & 0 & 0 & 0 \\
0 & 1 & 0 & 0 \\
0 & 0 & c_{34} & s_{34} \\
0 & 0 & -s_{34} & c_{34}
\end{array}\right), R_{24}\left(\theta_{24}\right)=\left(\begin{array}{cccc}
1 & 0 & 0 & 0 \\
0 & c_{24} & 0 & s_{24} \\
0 & 0 & 1 & 0 \\
0 & -s_{24} & 0 & c_{24}
\end{array}\right), \\
R_{14}\left(\theta_{14}\right)= & \left(\begin{array}{cccc}
c_{14} & 0 & 0 & s_{14} \\
0 & 1 & 0 & 0 \\
0 & 0 & 1 & 0 \\
-s_{14} & 0 & 0 & c_{14}
\end{array}\right), R_{12}\left(\theta_{12}\right)=\left(\begin{array}{cccc}
c_{12} & s_{12} & 0 & 0 \\
-s_{12} & c_{12} & 0 & 0 \\
0 & 0 & 1 & 0 \\
0 & 0 & 0 & 1
\end{array}\right), \\
R_{13}\left(\theta_{13}\right)= & \left(\begin{array}{cccc}
c_{13} & 0 & s_{13} & 0 \\
0 & 1 & 0 & 0 \\
-s_{13} & 0 & c_{13} & 0 \\
0 & 0 & 0 & 1
\end{array}\right), R_{23}\left(\theta_{23}\right)=\left(\begin{array}{cccc}
1 & 0 & 0 & 0 \\
0 & c_{23} & s_{23} & 0 \\
0 & -s_{23} & c_{23} & 0 \\
0 & 0 & 0 & 1
\end{array}\right) .
\end{aligned}
$$

Therefore $U_{(4 \times 4)}$ takes the form as

$$
\begin{aligned}
& U_{(4 \times 4)}=\left(\begin{array}{cccc}
c_{14} & 0 & 0 & s_{14} \\
-s_{14} s_{24} & c_{24} & 0 & c_{14} s_{24} \\
-c_{24} s_{14} s_{34} & -s_{24} s_{34} & c_{34} & c_{14} c_{24} s_{34} \\
-c_{24} s_{14} c_{34} & -s_{24} c_{34} & -s_{34} & c_{14} c_{24} c_{34}
\end{array}\right) \times\left(\begin{array}{cccc}
\tilde{U}_{e 1} & \tilde{U}_{e 2} & \tilde{U}_{e 3} & 0 \\
\tilde{U}_{\mu 1} & \tilde{U}_{\mu 2} & \tilde{U}_{\mu 3} & 0 \\
\tilde{U}_{\tau 1} & \tilde{U}_{\tau 2} & \tilde{U}_{\tau 3} & 0 \\
0 & 0 & 0 & 1
\end{array}\right) \\
& =\left(\begin{array}{cccc}
c_{14} \tilde{U}_{e 1} & c_{14} \tilde{U}_{e 2} & c_{14} \tilde{U}_{e 3} & s_{14} \\
-s_{14} s_{24} \tilde{U}_{e 1}+c_{24} \tilde{U}_{\mu 1} & -s_{14} s_{24} \tilde{U}_{e 2}+c_{24} \tilde{U}_{\mu 2} & -s_{14} s_{24} \tilde{U}_{e 3}+c_{24} \tilde{U}_{\mu 3} & c_{14} s_{24} \\
-c_{24} s_{14} s_{34} \tilde{U}_{e 1} & -c_{24} s_{14} s_{34} \tilde{U}_{e 2} & -c_{24} s_{14} s_{34} \tilde{U}_{e 3} & \\
-s 24 s 34 \tilde{U}_{\mu 1} & -s 24 s 34 \tilde{U}_{\mu 2} & -s 24 s 34 \tilde{U}_{\mu 3} & c_{14} c_{24} s_{34} \\
+c_{34} \tilde{U}_{\tau 1} & +c_{34} \tilde{U}_{\tau 2} & +c_{34} \tilde{U}_{\tau 3} & \\
-c_{24} c_{34} s_{14} \tilde{U}_{e 1} & -c_{24} c_{34} s_{14} \tilde{U}_{e 2} & -c_{24} c_{34} s_{14} \tilde{U}_{e 3} & \\
-s_{24} c_{34} \tilde{U}_{\mu 1} & -s_{24} c_{34} \tilde{U}_{\mu 2} & -s_{24} c_{34} \tilde{U}_{\mu 3} & c_{14} c_{24} c_{34} \\
-s_{34} \tilde{U}_{\tau 1} & -s_{34} \tilde{U}_{\tau 2} & -s_{34} \tilde{U}_{\tau 3} &
\end{array}\right)
\end{aligned}
$$


where $\tilde{U}_{\alpha i}$ etc. indicate the elements of the flavour mixing matrix in 3-flavour scenario, which can be expressed as $[9,16,17]$

$$
\tilde{U}=\left(\begin{array}{ccc}
c_{12} c_{13} & s_{12} s_{13} & s_{13} \\
-s_{12} c_{23}-c_{12} s_{23} s_{13} & c_{12} c_{23}-s_{12} s_{23} s_{13} & s_{23} c_{13} \\
s_{12} s_{23}-c_{12} c_{23} s_{13} & -c_{12} s_{23}-s_{12} c_{23} s_{13} & c_{23} c_{13}
\end{array}\right)
$$

In Eqs. (4-6), $\cos \theta_{i j}=c_{i j}$ and $\sin \theta_{i j}=s_{i j}$ where $\theta_{i j}$ defines the mixing angle between $i$ th and $j$ th neutrinos with mass eigenstates $\left|\nu_{i}\right\rangle$ and $\left|\nu_{j}\right\rangle$.

The time evolution equation in the case of four neutrino flavours, $\left|\nu_{e}\right\rangle,\left|\nu_{\mu}\right\rangle,\left|\nu_{\tau}\right\rangle$ and $\left|\nu_{s}\right\rangle$ is given by

$$
i \frac{d}{d t}\left(\begin{array}{c}
\nu_{e} \\
\nu_{\mu} \\
\nu_{\tau} \\
\nu_{s}
\end{array}\right)=H\left(\begin{array}{c}
\nu_{e} \\
\nu_{\mu} \\
\nu_{\tau} \\
\nu_{s}
\end{array}\right)
$$

where

$$
H=U_{(4 \times 4)} H_{d} U_{(4 \times 4)}^{\dagger} .
$$

In the above, the Hamiltonian in the mass basis is given by

$$
H_{d}=\left(\begin{array}{cccc}
E_{1} & 0 & 0 & 0 \\
0 & E_{2} & 0 & 0 \\
0 & 0 & E_{3} & 0 \\
0 & 0 & 0 & E_{4}
\end{array}\right)
$$

where $E_{i}, i=1-4$ are the energy eigenvalues which can be expressed in terms of the momentum $p$ and mass eigenvalues $m_{i}$, as

$$
E_{i}=\sqrt{p_{i}^{2}+m_{i}^{2}} \simeq p_{i}+\frac{m_{i}^{2}}{2 p_{i}} \simeq p+\frac{m_{i}^{2}}{2 E},
$$

with $i=1,2,3,4$ and $p_{i} \simeq p$. With this $H_{d}$ can be rewritten as

$$
H_{d}=\left(\begin{array}{cccc}
p & 0 & 0 & 0 \\
0 & p & 0 & 0 \\
0 & 0 & p & 0 \\
0 & 0 & 0 & p
\end{array}\right)+\frac{1}{2 E}\left(\begin{array}{cccc}
m_{1}^{2} & 0 & 0 & 0 \\
0 & m_{2}^{2} & 0 & 0 \\
0 & 0 & m_{3}^{2} & 0 \\
0 & 0 & 0 & m_{4}^{2}
\end{array}\right)
$$

In Eq. (11), the matrix $\operatorname{diag}(p, p, p, p)$ does not contribute to the neutrino oscillations as it does not induce any phase differences between the neutrinos and hence we do not consider 
this term further in the calculation. Subtracting $m_{1}^{2}$ from all the diagonal elements of the matrix $\operatorname{diag}\left(m_{1}^{2}, m_{2}^{2}, m_{3}^{2}, m_{4}^{2}\right)$, we have

$$
H_{d}=\frac{1}{2 E} \operatorname{diag}\left(0, \Delta m_{21}^{2}, \Delta m_{31}^{2}, \Delta m_{41}^{2}\right),
$$

where $\Delta m_{21}^{2}=m_{2}^{2}-m_{1}^{2}, \Delta m_{31}^{2}=m_{3}^{2}-m_{1}^{2}, \Delta m_{41}^{2}=m_{4}^{2}-m_{1}^{2}$.

As discussed in Section 1 the violation of equivalence principle can also induce neutrino oscillations due to different gravitational couplings to different types of neutrinos. As the neutrinos of different types couple differently, the gravitational constant $(G)$ should be different for different types of neutrinos. In addition to the mass induced oscillations, the gravity eigenstates $\left(\left|\nu_{G i}\right\rangle\right)$ can also lead to the neutrino oscillations if gravity eigenstates for neutrinos are not identical to their flavour eigenstates. We explore the mass flavour oscillations in matter and gravity induced oscillations in a single framework by considering $\left|\nu_{\alpha}\right\rangle \neq\left|\nu_{i}\right\rangle \neq\left|\nu_{G i}\right\rangle$. It is discussed in Section 1 that the neutrino energies are red-shifted by an amount $E \rightarrow E^{\prime}=\sqrt{g_{00}} E$ with respect to the vacuum with $E^{\prime}=E\left(1-\frac{G M}{R}\right)=E(1+\phi)$ where $g_{00}=(1+2 \phi)$, $\phi$ being the gravitational potential, $M$ is the mass of the source of the gravitational field and $R$ is the distance over which the gravitational field operates. In 4-flavour framework, the gravity eigenstates $\left|\nu_{G i}\right\rangle(i=1,2,3,4)$ are connected to the flavour eigenstates $\left|\nu_{\alpha}\right\rangle(\alpha=e, \mu, \tau, s)$ through a mixing matrix $U_{(4 \times 4)}^{\prime}$ with gravity-flavour mixing angle $\theta_{i j}^{G}(i \neq j), i, j=1,2,3,4$ in the presence of the gravitational field. Thus

$$
\left|\nu_{\alpha}\right\rangle=U_{(4 \times 4)}^{\prime}\left|\nu_{G_{i}}\right\rangle
$$

where the gravity-flavour mixing matrix $\left(U_{(4 \times 4)}^{\prime}\right)$ can be represented as

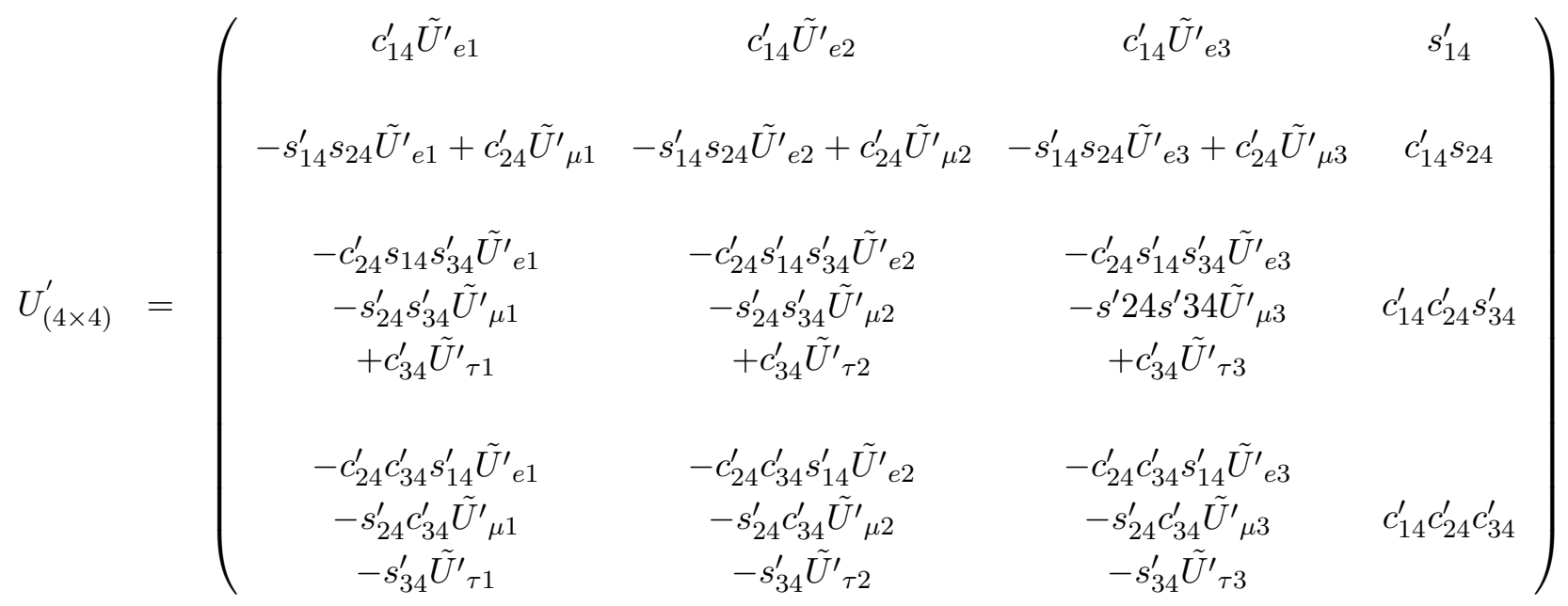

In the above, $\tilde{U}_{\alpha i}^{\prime}(\alpha=e, \mu, \tau ; i=1,2,3)$ are the elements of the 3-neutrino gravity-flavour mixing matrix $\tilde{U}^{\prime}$, whose term is similar to Eq. (6) (but the mixing angles may be different from mass-flavour case). As mentioned earlier, the gravity-flavour mixing angles are denoted 
by $\theta_{i j}^{G}$. It may be noted that Eq. (14) is similar to Eq. (5) where $\theta_{i j} \rightarrow \theta_{i j}^{G}, U_{(4 \times 4)} \rightarrow U_{(4 \times 4)}^{\prime}$, $\tilde{U} \rightarrow \tilde{U}^{\prime}$. Also note that, Eq. (13) is similar to Eq. (2) where $U_{(4 \times 4)} \rightarrow U_{(4 \times 4)}^{\prime}$ and $\left|\nu_{i}\right\rangle \rightarrow$ $\left|\nu_{G}\right\rangle$. The evolution equation in flavour basis due to the presence of the gravitational field (only for gravity-flavour oscillation case) is therefore written as

$$
i \frac{d}{d t}\left|\nu_{\alpha}\right\rangle=H^{\prime}\left|\nu_{\alpha}\right\rangle
$$

where $H^{\prime}=U_{(4 \times 4)}^{\prime} H_{G} U_{(4 \times 4)}^{\prime \dagger}$ and for 4-flavour scenario $H_{G}=\operatorname{diag}\left(E_{G 1}, E_{G 2}, E_{G 3}, E_{G 4}\right)$ (diagonal in gravity basis $i \frac{d}{d t}\left|\nu_{G}\right\rangle=H_{G}\left|\nu_{G}\right\rangle$ ). Substituting $E_{G 1}$ from other elements of $H_{G}, H_{G}$ takes the form

$$
H_{G}=\left(\begin{array}{cccc}
0 & 0 & 0 & 0 \\
0 & \Delta E_{21, G} & 0 & 0 \\
0 & 0 & \Delta E_{31, G} & 0 \\
0 & 0 & 0 & \Delta E_{41, G}
\end{array}\right) .
$$

If the equivalence principle is indeed violated, all the gravitational energy eigenvalues will induce phase differences to neutrino eigenstates and therefore we have

$$
\left.H_{G}=\operatorname{diag}\left[\left(1-\phi \alpha_{1}\right) E,\left(1-\phi \alpha_{2}\right) E,\left(1-\phi \alpha_{3}\right) E,\left(1-\phi \alpha_{4}\right) E\right)\right] .
$$

With $\phi \alpha_{i}=\frac{G_{i} M}{R}=\frac{G M}{R} \alpha_{i}$. In this case, the phase differences can be expressed as

$$
\Delta E_{i j, G}=\frac{G M}{R} \Delta \alpha_{i j} E=\frac{G M}{R}\left(\alpha_{i}-\alpha_{j}\right) E=\phi \Delta \alpha_{i j} E=\Delta f_{i j} E,
$$

where $\Delta f_{i j}=\frac{G M}{R} \Delta \alpha_{i j}=\Delta \alpha_{i j} \phi ; i, j=1,2,3,4$. After substracting $\left(1-\phi \alpha_{1}\right) E$ term from all the diagonal elements of $H_{G}$, we have $H_{G}=\operatorname{diag}\left(0, \Delta f_{21} E, \Delta f_{31} E, \Delta f_{41} E\right)$. It is now well established that neutrino oscillations in matter may differ significantly from that in vacuum and which was first observed by Mikheyev - Smirnov - Wolfenstein [18, 19], and known as MSW effect. In the present neutrino oscillations formalism we also include the MSW effect. The effective Hamiltonian of the system in flavour basis including both gravity effect and matter effect is given by

$$
\begin{aligned}
H^{\prime \prime} & =H+H^{\prime}+V \\
& =U_{(4 \times 4)} H_{d} U_{(4 \times 4)}^{\dagger}+U_{(4 \times 4)}^{\prime} H_{G} U_{(4 \times 4)}^{\prime \dagger}+V,
\end{aligned}
$$

such that, $i \frac{d}{d t}\left|\nu_{\alpha}\right\rangle=H^{\prime \prime}\left|\nu_{\alpha}\right\rangle$. In the above, the matter potential $(V)$ can be written as

$$
V=\operatorname{diag}\left(V_{C C}, 0,0,-V_{N C}\right)
$$

where $V_{C C}$ is the charged current potential that appears due to the interactions with the electrons of the medium, which are mediated by the $W^{ \pm}$exchange and $V_{N C}$ denotes the 
neutral current potential responsible for the interactions mediated by $Z^{0}$ bosons. With $V_{C C}=\sqrt{2} G_{F} N_{e}$ and $V_{N C}=\frac{G_{F} N_{n}}{\sqrt{2}}$, the matter potential $(V)$ can be expressed as

$$
V=\operatorname{diag}\left(\sqrt{2} G_{F} N_{e}, 0,0, G_{F} N_{n} / \sqrt{2}\right)
$$

where $G_{F}$ is the Fermi constant, $N_{e}$ and $N_{n}$ are the number densities of electron and neutrons respectively inside the matter through which neutrinos propagate. In our formalism, for the purpose of the calculation we assume that the mass mixing angles $\left(\theta_{i j}\right)$ and gravity mixing angles $\left(\theta_{i j}^{G}\right)$ with the flavour eigenstates are same, and hence $U_{(4 \times 4)}=U_{(4 \times 4)}^{\prime}=U$. The effective Hamiltonian according to this assumption takes the form

$$
\begin{aligned}
H^{\prime \prime}= & U\left(H_{d}+H_{G}\right) U^{\dagger}+V \\
= & U\left(\operatorname{diag}\left(0, \frac{\Delta m_{21}^{2}}{2 E}, \frac{\Delta m_{31}^{2}}{2 E}, \frac{\Delta m_{41}^{2}}{2 E}\right)\right. \\
& \left.+\operatorname{diag}\left(0, \Delta f_{21} E, \Delta f_{31} E, \Delta f_{41} E\right)\right) U^{\dagger}+V .
\end{aligned}
$$

We neglect the terms $\Delta m_{21}^{2}$ and $\Delta f_{21}$ by assuming that the neutrino mass eigenstates $\left|\nu_{1}\right\rangle$, $\left|\nu_{2}\right\rangle$ as well as gravity eigenstates $\left|\nu_{G 1}\right\rangle,\left|\nu_{G 2}\right\rangle$ are very close to each other. Thus the above Eq. (21) can be written as

$$
\begin{aligned}
H^{\prime \prime} & =U \operatorname{diag}\left(0,0, \frac{\Delta m_{31}^{2}}{2 E}+\Delta f_{31} E, \frac{\Delta m_{41}^{2}}{2 E}+\Delta f_{41} E\right) U^{\dagger}+V \\
& =U \operatorname{diag}\left(0,0, \frac{\Delta \mu_{31}^{2}}{2 E}, \frac{\Delta \mu_{41}^{2}}{2 E}\right) U^{\dagger}+V
\end{aligned}
$$

In the above,

$$
\begin{aligned}
\frac{\Delta \mu_{31}^{2}}{2 E} & =\frac{\Delta m_{31}^{2}}{2 E}+\Delta f_{31} E, \\
\frac{\Delta \mu_{41}^{2}}{2 E} & =\frac{\Delta m_{41}^{2}}{2 E}+\Delta f_{41} E .
\end{aligned}
$$

In Eq. (22), the unitary matrix $U$ is the $4 \times 4$ matrix similar to that given in Eq. (5) (with different mixing angle parameters). The active neutrino mixing angles described in Eq. (6) are obtained from the latest bounds given by different neutrino experiments [20]. In this work, we use the best fit values of the standard three neutrino oscillation parameters which are given as [20]

$$
\begin{array}{r}
\theta_{12}=33.96^{0}, \quad \theta_{23}=48.3^{0}, \quad \theta_{13}=8.61^{0} \\
\Delta m_{21}^{2}=7.53 \times 10^{-5} \mathrm{eV}^{-2}, \quad \Delta m_{31}^{2}=2.5 \times 10^{-3} \mathrm{eV}^{-2} .
\end{array}
$$

For simplicity, we also consider the case of normal hierarchy for neutrino eigenstates and $\mathrm{CP}$ violating phase $\delta_{\mathrm{CP}}=0$.

Apart from the active neutrino oscillation parameters mentioned in Eq. (24), there are three active-sterile neutrino mixing angles $\theta_{14}, \theta_{24}, \theta_{34}$. Several neutrino oscillation 
experiments such as MINOS [21]-[32], Daya Bay [33]-[40], Bugey [41], T2K [42], IceCube [43] etc. provide stringent limits on these mixing angles $\left(\theta_{i 4}, i=1-3\right)$ for different values of mass square difference $\Delta m_{41}^{2}$. We use the combined limit on mixing angle $\theta_{14}$ obtained from the analyses by Daya Bay, MINOS and Bugey-3 [33]. However, in a recent work by Adams et al., constraints from cosmological data were also taken into account [44] along with the neutrino oscillation results from other experiments. From their analyses, they conclude that Planck data exclude the regions with $\Delta m_{41}^{2} \geq 5 \times 10^{-2} \mathrm{eV}^{2}$ and for $\Delta m_{41}^{2} \leq 5 \times 10^{-2}$ $\mathrm{eV}^{2}$, limits from the combined analysis of Daya Bay, MINOS and Bugey-3 [33] become significant (see Fig. 2 and Fig. 4 of Ref. [44] for details). Using these constraints, in the present work, we adopt $\theta_{14}=3.6^{0}$ and present our results for two values of $\Delta m_{41}^{2}$ namely, $\Delta m_{41}^{2}=1 \times 10^{-3} \mathrm{eV}^{2}$ and $\Delta m_{41}^{2}=3 \times 10^{-3} \mathrm{eV}^{2}$, consistent with the latest experimental findings. MINOS and MINOS+ $[23,32]$ also provide limits on the active-sterile mixing angle $\theta_{24}$. From their analyses, it is found that for $\Delta m_{41}^{2} \geq 10^{-2} \mathrm{eV}^{2}$, MINOS+ provides strong upper bound on the mixing angle $\theta_{24}$. However, it is observed that $\theta_{24} \leq 26.7^{0}$ with the choice $\Delta m_{41}^{2}=1 \times 10^{-3} \mathrm{eV}^{2}$ and $\theta_{24} \leq 50.7^{0}$ when $\Delta m_{41}^{2}=3 \times 10^{-3} \mathrm{eV}^{2}$. However, recent analysis for the search of sterile neutrino performed by T2K far detector with $295 \mathrm{~km}$ baseline length [42] predicts $\theta_{24} \leq 22.7^{0}$ for $\Delta m_{41}^{2}=1 \times 10^{-3} \mathrm{eV}^{2}$ and for $\Delta m_{41}^{2}=3 \times 10^{-3} \mathrm{eV}^{2}$ the limit on mixing angle is $\theta_{24} \leq 15.3^{0}$. With the above limit on $\theta_{24}$ for $\Delta m_{41}^{2}=3 \times 10^{-3} \mathrm{eV}^{2}$, the limit on $\theta_{34}$ is found to be $\theta_{34} \leq 53.1^{0}$ (see Fig. 4 of Ref. [42] for details). Therefore, we observe that for smaller values of $\Delta m_{41}^{2} \sim 10^{-3} \mathrm{eV}^{2}$, although the mixing angle $\theta_{14}$ is very much constrained, limits on other mixing angles namely $\theta_{24}, \theta_{34}$ are not much stringent. In this work, we adopt two different sets of active-sterile neutrino mixing angles given in Table 1 which are in agreement with different neutrino oscillation experimental results for smaller values of $\Delta m_{41}^{2} \sim 10^{-3} \mathrm{eV}^{2}$.

It is to be noted that apart from the $\delta_{C P}$, the $3+1$ scenario involves two new CP phases $\delta_{14}$ and $\delta_{24}$. However, as mentioned in [33], experiments like MINOS, Daya Bay and Bugey-3 are based on disappearance measurements which makes them insensitive to $\mathrm{CP}$ phases. Apart from that, IceCube [43] experiment also carry out their measurements of active-sterile mixing angle considering $\mathrm{CP}$ phases to be zero. We use various limits on active-sterile mixing provided by these experiments that are insensitive to the choice of $\mathrm{CP}$ phases. In the present work we assume a CP conserving scenario and set all CP phases to zero.

With the two sets of mixing angles tabulated in Table 1 and neutrino mass square differences mentioned above, we now calculate the modified four neutrino oscillations probabilities within matter including the effects of violation of equivalence principle with new VEP parameters $\Delta f_{31}$ and $\Delta f_{41}$. The Hamiltonian $H^{\prime \prime}$ is then diagonalised by a new $4 \times 4$ unitary matrix $U^{m}$ whose elements are similar to that of the matrix $U$ (as in Eq. 5 ) but with new modified mixing angles. Therefore, the oscillation probability for a neutrino $\left|\nu_{\alpha}\right\rangle$ 
Table 1: Chosen 4-flavour mixing angle parameter sets for the calculation of gravity induced 4-flavour oscillations in matter.

\begin{tabular}{|c|c|c|c|}
\hline Set & $\theta_{14}$ & $\theta_{24}$ & $\theta_{34}$ \\
\hline 1 & $3.6^{\circ}$ & $4.0^{\circ}$ & $18.48^{\circ}$ \\
\hline 2 & $2.5^{\circ}$ & $10.0^{\circ}$ & $30.0^{\circ}$ \\
\hline
\end{tabular}

having flavour $\alpha$ oscillate to a neutrino $\left|\nu_{\beta}\right\rangle$ of flavour $\beta$ is given by the expression $[45,46]$

$$
P_{\alpha \beta}=\delta_{\alpha \beta}-4 \sum_{j>i} U_{\alpha i}^{m} U_{\beta i}^{m} U_{\alpha j}^{m} U_{\beta j}^{m} \sin ^{2}\left(\frac{\pi L}{\lambda_{i j}}\right),
$$

where $U_{\alpha i}^{m}$ etc. are the matrix elements of the unitary matrix $\left(U^{m}\right)$, which is computationally obtained by diagonalising the effective Hamiltonian $H^{\prime \prime}$ in Eq. (22) and $L$ indicates the baseline length. The oscillation length $\left(\lambda_{i j}\right)$ in the presence of both mass and gravity induced oscillations in matter can be expressed as

$$
\lambda_{i j}=\frac{2 \pi}{E_{j}^{\prime}-E_{i}^{\prime}}=\frac{2 \pi}{\Delta E_{i j}^{\prime}}
$$

where $E_{i}^{\prime}, E_{j}^{\prime}(i, j=1,2,3,4 ; i \neq j)$ are the eigenvalues of the effective Hamiltonian $H^{\prime \prime}$ (Eq. (22)). Since the mass eigenstates $\left|\nu_{1}\right\rangle$ and $\left|\nu_{2}\right\rangle$ can be assumed to be almost degenerate $\left(\Delta m_{21}^{2} \sim 10^{-5} \mathrm{eV}^{2}\right)$, we have $\Delta m_{31}^{2} \simeq \Delta m_{32}^{2}, \Delta m_{41}^{2} \simeq \Delta m_{42}^{2}$. We follow similar convention for neutrino gravity eigenstates, such that $\left|\nu_{G 1}\right\rangle$ and $\left|\nu_{G 2}\right\rangle$ are also almost degenerate $\left(\Delta f_{21}=0\right)$ and adopt $\Delta f_{31} \simeq \Delta f_{32}$ and $\Delta f_{41} \simeq \Delta f_{42}$. In the following, we explicitly furnish the expressions for 4-neutrino oscillation probabilities for the cases of $\nu_{\mu} \rightarrow \nu_{\mu}$ and $\nu_{e} \rightarrow \nu_{\mu}$ oscillations.

$$
\begin{aligned}
P_{\mu \mu}^{4}= & 1-4\left[\left|U_{\mu 1}^{m}\right|^{2}\left|U_{\mu 2}^{m}\right|^{2} \sin ^{2}\left(\frac{\Delta E_{12}^{\prime} L}{2}\right)+\left|U_{\mu 1}^{m}\right|^{2}\left|U_{\mu 3}^{m}\right|^{2} \sin ^{2}\left(\frac{\Delta E_{13}^{\prime} L}{2}\right)+\right. \\
& \left|U_{\mu 1}^{m}\right|^{2}\left|U_{\mu 4}^{m}\right|^{2} \sin ^{2}\left(\frac{\Delta E_{14}^{\prime} L}{2}\right)+\left|U_{\mu 2}^{m}\right|^{2}\left|U_{\mu 3}^{m}\right|^{2} \sin ^{2}\left(\frac{\Delta E_{23}^{\prime} L}{2}\right)+ \\
& \left.\left|U_{\mu 2}^{m}\right|^{2}\left|U_{\mu 4}^{m}\right|^{2} \sin ^{2}\left(\frac{\Delta E_{24}^{\prime} L}{2}\right)+\left|U_{\mu 3}^{m}\right|^{2}\left|U_{\mu 4}^{m}\right|^{2} \sin ^{2}\left(\frac{\Delta E_{34}^{\prime} L}{2}\right)\right] \\
P_{e \mu}^{4}= & 4\left[\left|U_{e 1}^{m}\right|\left|U_{\mu 1}^{m}\right|\left|U_{e 2}^{m}\right|\left|U_{\mu 2}^{m}\right| \sin ^{2}\left(\frac{\Delta E_{12}^{\prime} L}{2}\right)+\left|U_{e 1}^{m}\right|\left|U_{\mu 1}^{m}\right|\left|U_{e 3}^{m}\right|\left|U_{\mu 3}^{m}\right| \sin ^{2}\left(\frac{\Delta E_{13}^{\prime} L}{2}\right)+\right. \\
& \left.\left|U_{e 1}^{m}\right|\left|U_{\mu 1}^{m}\right|\left|U_{e 4}^{m}\right|\left|U_{\mu 4}^{m}\right|\right) \sin ^{2}\left(\frac{\Delta E_{14}^{\prime} L}{2}\right)+\left|U_{e 2}^{m}\right|\left|U_{\mu 2}^{m}\right|\left|U_{e 3}^{m}\right|\left|U_{\mu 3}^{m}\right| \sin ^{2}\left(\frac{\Delta E_{23}^{\prime} L}{2}\right)+ \\
& \left.\left|U_{e 2}^{m}\right|\left|U_{\mu 2}^{m}\right|\left|U_{e 4}^{m}\right|\left|U_{\mu 4}^{m}\right| \sin ^{2}\left(\frac{\Delta E_{24}^{\prime} L}{2}\right)+\left|U_{e 3}^{m}\right|\left|U_{\mu 3}^{m}\right|\left|U_{e 4}^{m}\right|\left|U_{\mu 4}^{m}\right| \sin ^{2}\left(\frac{\Delta E_{34}^{\prime} L}{2}\right)\right]
\end{aligned}
$$


The expressions for other probabilities can similarly be written. It is to be noted that, earlier studies of violation of equivalence principle with IceCube neutrino data by Esmaili et. al set a stringent bound on $\Delta f_{31} \leq 7 \times 10^{-27}$ [47]. Therefore, in the present work, we consider $\Delta f_{31}=5 \times 10^{-27}$ which is in agreement with previous analysis. However, since the value of $\Delta f_{31}$ is negligibly small, we do not expect any significant VEP effect due to $\Delta f_{31}$. On the other hand there exists no bound on the VEP associated with the sterile neutrino $\Delta f_{41}$. In the next section, we investigate how VEP induced by the sterile neutrino affects the four flavour neutrino oscillations in matter.

\section{Calculations and Results}

\subsection{Gravity-induced neutrino oscillations in matter}

In this section, we study modification of neutrino oscillation probabilities in matter in presence of possible violation of equivalence principle. For this purpose, we consider a 4flavour neutrino scenario, where the usual three families of active neutrinos $\left(\nu_{e}, \nu_{\mu}, \nu_{\tau}\right)$ are extended by an extra setrile neutrino $\left(\nu_{s}\right)$. In order to estimate the effect of the gravity induced oscillations, we consider baseline neutrino oscillations, where the neutrinos are produced from a neutrino factory or an accelerator and propagate from the source to a far terrestrial away detector through the Earth matter.

The probabilities of oscillations from one flavour to the other in the present framework are therefore an important component for the estimation of the neutrino flux at the end detector. Therefore we calculate the oscillation probabilities from one neutrino flavour to the other for the present 4-neutrino scenario where both the mass induced and gravity induced oscillations are considered. The probabilities are computed using the Eqs. (18) - (27). We demonstrate in this section how the nature of the probabilities are varied by the combined effect of the gravity induced factors as well as the mass-flavour oscillations in matter. For the present calculations we have chosen a demonstrative baseline length of $7000 \mathrm{Km}$ and the mean Earth matter density to be $4.15 \mathrm{gm} / \mathrm{cc}$. From Eq. (25) and Eq. (27) it is clear that the oscillatory part of the probability equations are controlled by the phase factor $\Delta E_{i j}^{\prime} L / 2$, where $\Delta E_{i j}^{\prime}$ are the difference of the eigenvalues $E_{i}^{\prime}$ and $E_{j}^{\prime}$ of the eigenstates designated by $i$ and $j$ respectively. In this work, the eigenvalues $E_{i}^{\prime}, E_{j}^{\prime}$ etc. are computationally obtained by explicitly diagonalising the Hamiltonian $H^{\prime \prime}$ (Eq. (22)) that includes both the mass induced effects, matter effects as also the gravity induced effects.

In Figs. 1-2, we show how the oscillation probabilities vary for the variation of gravity induced effects (designated by $\Delta f_{i j}$ ), when the mass square difference $\Delta m_{i j}^{2}$ are kept fixed. Since we are considering here a 4 -flavour scenario, these probability plots also demonstrate the 4-flavour oscillations considering both the mass flavour effects in matter and the gravity 

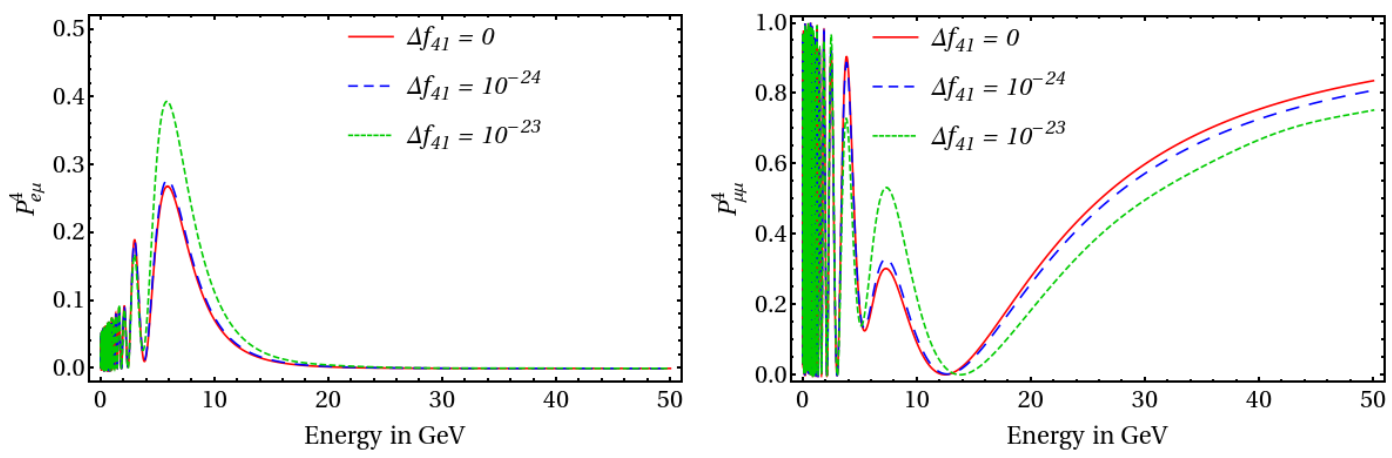

(a) Variation of $P_{e \mu}^{4}$ for $\Delta m_{41}^{2}=1 \times 10^{-3} \mathrm{eV}^{2}$

(b) Variation of $P_{\mu \mu}^{4}$ for $\Delta m_{41}^{2}=1 \times 10^{-3} \mathrm{eV}^{2}$
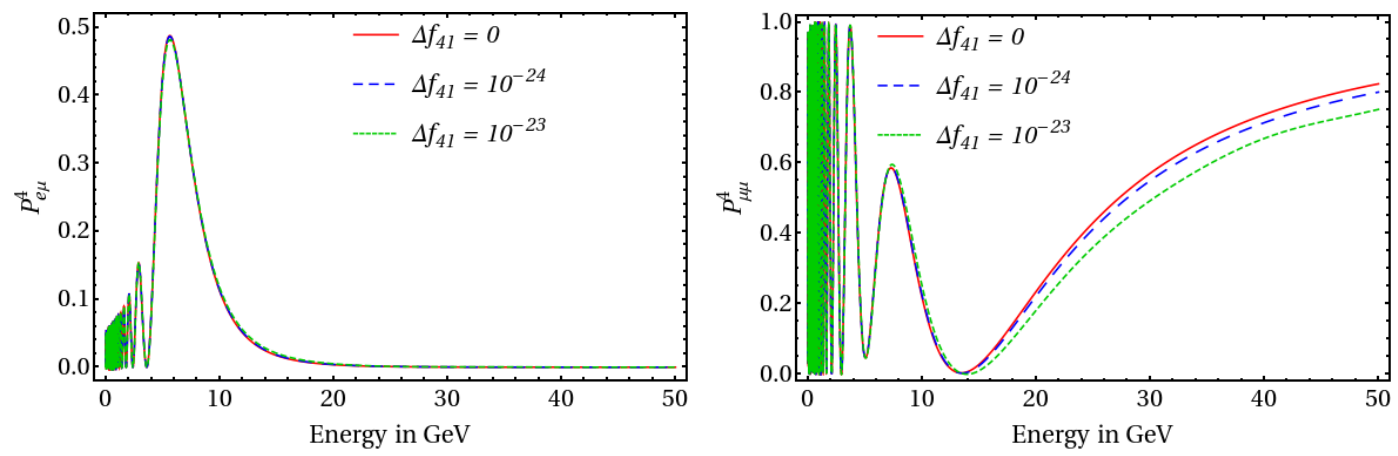

(c) Variation of $P_{e \mu}^{4}$ for $\Delta m_{41}^{2}=3 \times 10^{-3} \mathrm{eV}^{2}$

(d) Variation of $P_{\mu \mu}^{4}$ for $\Delta m_{41}^{2}=3 \times 10^{-3} \mathrm{eV}^{2}$

Figure 1: Neutrino oscillation probabilities in matter for a fixed value of $\Delta f_{31}=$ $5 \times 10^{-27}$ and for Set-1 with baseline length $L=7000 \mathrm{~km}$.

induced effects.

Figs. 1-2 show the variations of the probability $P_{e \mu}^{4}$ and $P_{\mu \mu}^{4}$ with different neutrino energies for different fixed chosen values of $\Delta f_{41}$. Three values of $\Delta f_{41}$ are chosen namely $\Delta f_{41}=0,10^{-24}, 10^{-23}$. Needless to mention that $\Delta f_{41}=0$. Fig. 1 shows the results obtained using the parameter for Set-1 whereas, in Fig. 2 the probability plots for Set-2 are shown. In both Fig. 1 and Fig. 2, the upper panels correspond to $\Delta m_{41}^{2}=1 \times 10^{-3}$ $\mathrm{eV}^{2}$, while for the lower panels $\Delta m_{41}^{2}=3 \times 10^{-3} \mathrm{eV}^{2}$. All the computations are made by adopting a fixed value of $\Delta f_{31}=5 \times 10^{-27}$. Similar plots for other probabilities (e.g. $P_{e e}^{4}$, $P_{e \tau}^{4}, P_{\mu \tau}^{4}$ etc.) can also be computed.

It is to be noted from Figs. 1-2, that the VEP effect is more prominent when $\Delta f_{41}=$ $10^{-23}$ and $\Delta m_{41}^{2}=1 \times 10^{-3} \mathrm{eV}^{2}$. It can also be noted that, if $\Delta m_{41}^{2}=1 \times 10^{-3} \mathrm{eV}^{2}$, the variations for $P_{e \mu}^{4}$ with $\Delta f_{41}=10^{-23}$ can be more than $50 \%$ for other chosen values of $\Delta f_{41}$ when Set-2 is adopted. Similar trends are also observed for $P_{\mu \mu}^{4}$. In the later case, the oscillation is more prominent for Set-2 than Set- 1 when $\Delta f_{41}=10^{-23}$ and $\Delta m_{41}^{2}=1 \times 10^{-3}$ $\mathrm{eV}^{2}$ are chosen. It is to be mentioned that all plots of Figs. 1, 2, the base length $L$ is chosen 

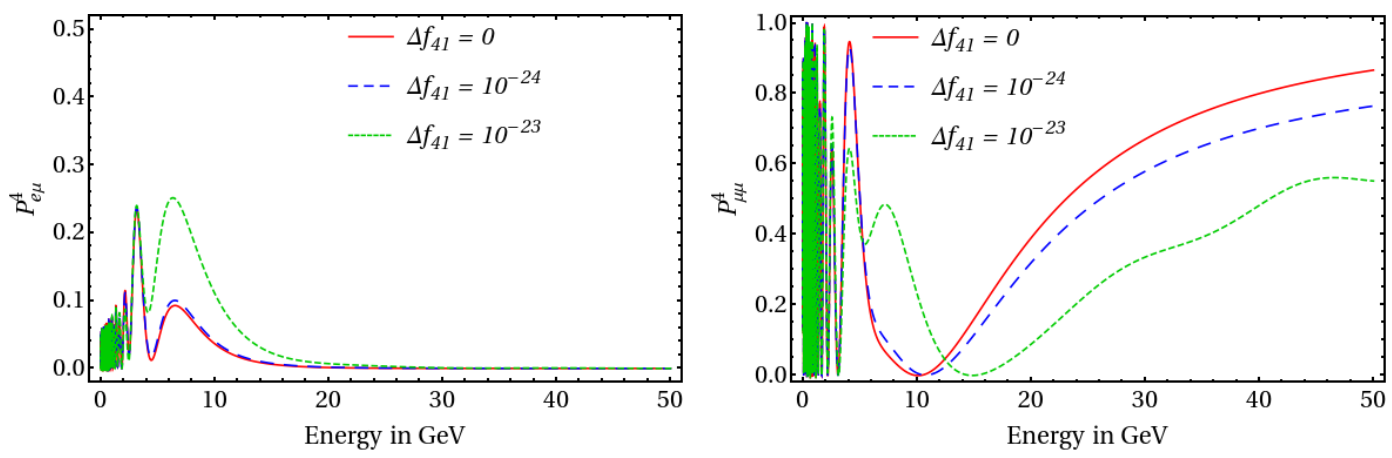

(a) Variation of $P_{e \mu}^{4}$ for $\Delta m_{41}^{2}=1 \times 10^{-3} \mathrm{eV}^{2}$

(b) Variation of $P_{\mu \mu}^{4}$ for $\Delta m_{41}^{2}=1 \times 10^{-3} \mathrm{eV}^{2}$
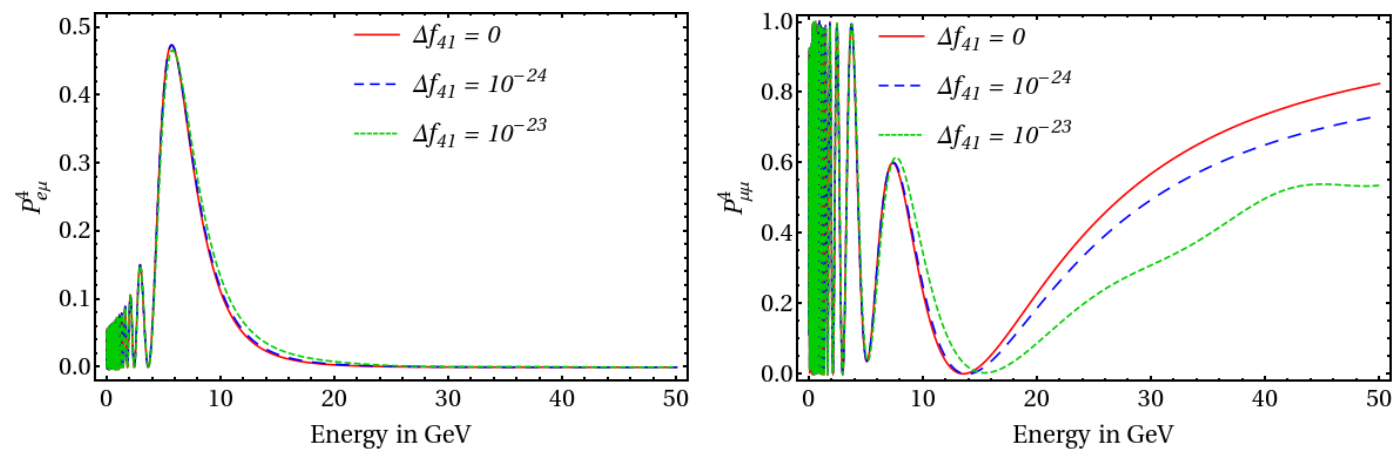

(c) Variation of $P_{e \mu}^{4}$ for $\Delta m_{41}^{2}=3 \times 10^{-3} \mathrm{eV}^{2}$

(d) Variation of $P_{\mu \mu}^{4}$ for $\Delta m_{41}^{2}=3 \times 10^{-3} \mathrm{eV}^{2}$

Figure 2: Neutrino oscillation probabilities in matter for a fixed value of $\Delta f_{31}=$ $5 \times 10^{-27}$ and for Set-2 with baseline length $L=7000 \mathrm{~km}$.

to be $7000 \mathrm{~km}$. Thus it is demonstrated from Figs. 1-2 that the probabilities are most affected by the gravity induced effects when $\Delta f_{41}=10^{-23}$.

\subsection{Effect of Gravity Induced Oscillation on a Long Baseline Neutrino Experiment}

In this section we pursue the effects of gravity induced oscillations on neutrino induced muon yields in long baseline (LBL) experiments. In a long baseline neutrino experiment, pions are initially produced in neutrino factories by directing a proton beam incident on a target. Pions decay into muons which suffer further decay in a muon storage ring producing neutrinos. Neutrinos are generated from 3-body decay of muons as ${ }^{7}$

$$
\begin{aligned}
& \mu^{-} \rightarrow e^{-}+\bar{\nu}_{e}+\nu_{\mu}, \\
& \mu^{+} \rightarrow e^{+}+\nu_{e}+\bar{\nu}_{\mu} .
\end{aligned}
$$

\footnotetext{
${ }^{7}$ Instead of the muon storage ring $\beta$ beams from $\beta$-decay of nucleons can also be treated as the source of neutrinos. In this case one should consider the channels $P_{\nu_{e} \rightarrow \nu_{x}}$, where $x=e, \mu, \tau$.
} 
Neutrinos produced in neutrino factory are then directed towards a neutrino detector far away from the source of the neutrinos and traverse through Earth matter to reach the detector. The muon neutrinos $\left(\nu_{\mu}, \bar{\nu}_{\mu}\right)$ generated in neutrino factory will suffer oscillations due to its passage through the Earth matter along the baseline. The $\nu_{\mu}\left(\bar{\nu}_{\mu}\right)$ will produce $\mu^{-}\left(\mu^{+}\right)$at the detector by charged current (CC) interaction with the detector material. If it is pure $\mu^{-}$at the source then only $\nu_{\mu}$ beam will propagate along the baseline and $\mu^{-}$will be produced at the detector end which the latter would detect. Those muons are called right sign muon. Needless to mention that $\nu_{\mu}$ flux at the source will suffer depletion due to the oscillation and same will happen to the muon yield. However if the detector detects a $\mu^{+}$ instead, then it must be that $\bar{\nu}_{\mu}$ reaches the detector and $\bar{\nu}_{\mu}$ can only be created in the beam (produced by the decay of $\mu^{-}$) through the oscillation $\bar{\nu}_{e} \rightarrow \bar{\nu}_{\mu}$ during the passage of $\bar{\nu}_{e}$ through the baseline. These events are termed as wrong sign muon events. The situation is just reversed if $\bar{\nu}_{\mu}$ beam is produced at the storage ring from the decay of $\mu^{+}$. But the right sign and the wrong sign muon events can be distinguished by a iron calorimeter detector (such as the one considered for the present work) when the ICAL detector is magnetized.

For this purpose, we need to compute the probabilities $P_{\bar{e} \bar{\mu}}^{4}$, where $P_{\bar{e} \bar{\mu}}^{4}$ is the $\nu_{\bar{e}}$ to $\nu_{\bar{\mu}}$ oscillation probability. It is to be noted that, for the case of anti-neutrinos the matter potential $V$ (see earlier) changes sign. Therefore the computation of $P_{\bar{e} \bar{\mu}}^{4}$ is done by considering $V$ to $-V$ in the relevant equations described in sect. 2 .

As mentioned above, we first consider the neutrino (anti-neutrino) flux in the neutrino factory which is expressed as [48, 49]

$$
\frac{d^{2} \Phi_{\nu_{\mu}, \bar{\nu}_{\mu}}}{d y d A}=\frac{4 n_{\mu}}{\pi L^{2} m_{\mu}^{6}} E_{\mu}^{4} y^{2}(1-\beta)\left[3 m_{\mu}^{2}-4 E_{\mu}^{2} y(1-\beta)\right]
$$

and similarly $\nu_{e}\left(\bar{\nu}_{e}\right)$ flux is given by

$$
\frac{d^{2} \Phi_{\nu_{e}, \bar{\nu}_{e}}}{d y d A}=\frac{24 n_{\mu}}{\pi L^{2} m_{\mu}^{6}} E_{\mu}^{4} y^{2}(1-\beta)\left[m_{\mu}^{2}-2 E_{\mu}^{2} y(1-\beta)\right] .
$$

where different terms are given as follows

- $E_{\mu}$ : muon energy

- $n_{\mu}$ : number of injected muons

- $L$ : distance between neutrino factory and the end detector (baseline length)

- $y=\frac{E_{\nu}}{E_{\mu}}$ where $E_{\nu}$ is energy of neutrino

- $\beta$ is the boost factor 
It is to be noted that the expressions for neutrino fluxes in Eqs. (29-30) are derived under the following approximations; i) neutrinos are not polarised and ii) the angle between direction of neutrino beam towards the detector and the beam axis is assumed to be zero. For the computations of neutrino flux using Eqs. $(28,29)$, we consider $\sim 10^{21}$ protons on target per year and muon injection energy of $50 \mathrm{GeV}$. In Fig. 3 we show the flux for $\nu_{\mu}$ and $\overline{\nu_{e}}$.

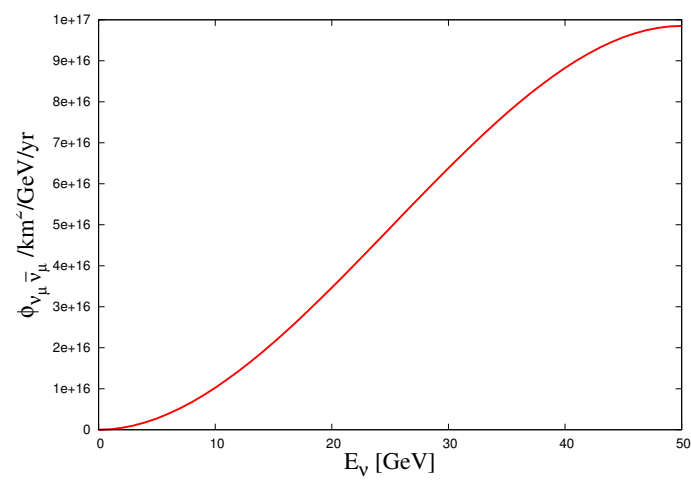

(a)

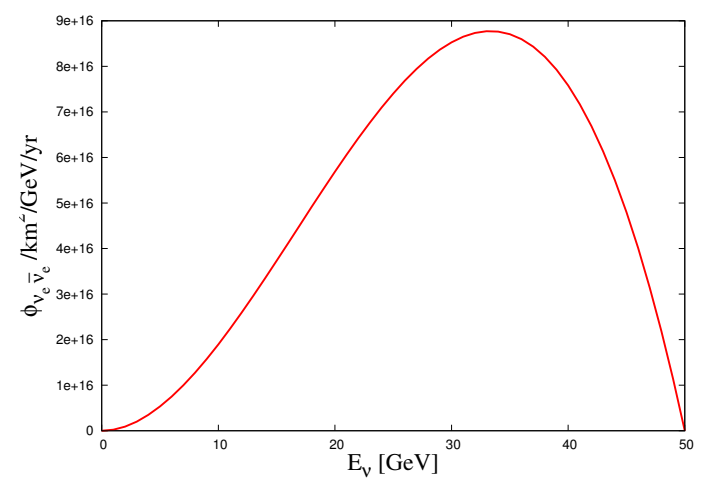

(b)

Figure 3: Flux of (a) $\nu_{\mu}$ and (b) $\overline{\nu_{e}}$ for muon decay at muon storage ring with muon injection energy of $50 \mathrm{GeV}$. See text for details.

As mentioned, in this work we consider the detector to be a magnetized iron calorimeter (ICAL) detector. Such a detector of 50 kTon mass has been suggested for the proposed India-based Neutrino Observatory (INO) [10]. The proposed ICAL detector at INO [10] is basically a stack of 151 iron plates of thickness $5.6 \mathrm{~cm}$ and each plates are separated by a gap of $4 \mathrm{~cm}$ containing a total of $50 \mathrm{kTon}$ of detector iron. Here we consider a baseline length of $7359 \mathrm{~km}$ which is roughly the distance between CERN and proposed INO site. Beam of $\nu_{\mu}\left(\bar{\nu}_{\mu}\right)$ from a neutrino factory after reaching such a magnetized ICAL detector will undergo charged current interactions and produce $\mu^{-}\left(\mu^{+}\right)$which form muon tracks while passing through different layers of the detector of different curvature due to magnetic field. Observing the direction and curvature of the muon tracks one can distinguish the right sign and wrong sign muons inside detector. As mentioned, the flux of neutrino (anti-neutrino) beam will undergo gravity induced and mass induced four flavour oscillations in matter before reaching the detector. Thus the neutrino (anti-neutrino) flux at the detector will be rescaled by the corresponding probabilities. For $\nu_{\mu}$ and $\bar{\nu}_{e}$ beam (produced from the decay $\mu^{-}$at the storage ring) if $\mu^{+}$is registered in ICAL then this is referred to as appearance channel since it originates due the oscillation $\bar{\nu}_{e} \rightarrow \bar{\nu}_{\mu}$ while for the same beam $\mu^{-}$track is considered as disappearance channel as $\nu_{\mu}$ disappears via the oscillation $\nu_{\mu} \rightarrow \nu_{x}, x \neq \mu$. In this section, we present the expected yield of right sign muon $\left(\mu^{-}\right)$and wrong sign muon $\left(\mu^{+}\right)$at ICAL detector in presence of gravity induced neutrino oscillations in four flavour 
scenario.

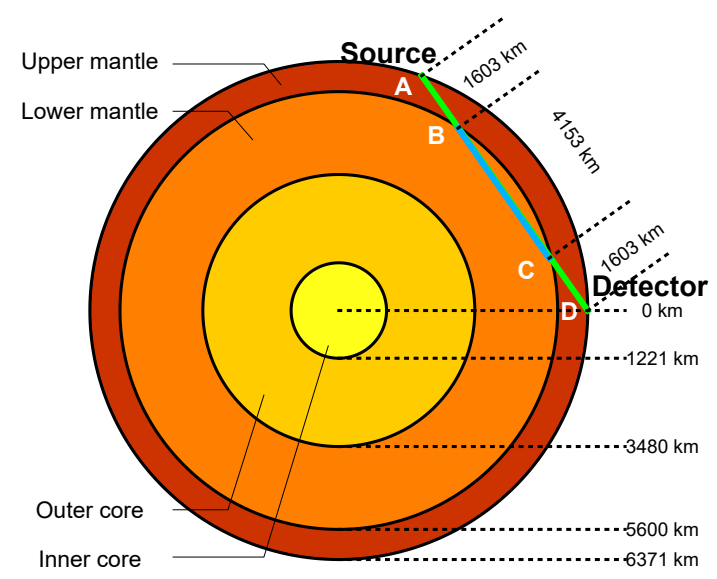

Figure 4: Different layers of earth and projected travel path of neutrinos for long basline neutrino detector placed at $7359 \mathrm{~km}$ from source.

Different neutrino oscillation probabilities calculated in previous section are presented assuming average Earth density $\rho=4.15 \mathrm{gm} / \mathrm{cc}$. However for the present scenario, where the baseline length $\sim 7359 \mathrm{~km}$, neutrinos travel through Earth crust and mantle. Width of Earth crust is very small $\sim 10-15 \mathrm{~km}$ compared to the long baseline oscillation length 7359 $\mathrm{km}$. Therefore, oscillation effects due to crust can be ignored safely and we consider neutrino oscillation through upper and lower mantle only, as demonstrated in Fig. 4. Density of upper (lower) mantle of Earth is $\rho_{u p}=3.9 \mathrm{gm} / \mathrm{cc}\left(\rho_{\text {low }}=4.5 \mathrm{gm} / \mathrm{cc}\right)$ [50]. From Fig. 4, we observe that for the chosen long baseline length, neutrinos traverse through upper mantle initially for first $1603 \mathrm{kms}$ and then enters lower mantle where it travels a distance of $4153 \mathrm{kms}$ and finally enters again into the upper mantle through which they travel another $1603 \mathrm{kms}$ to reach the detector. We use the above two layer formalism to calculate neutrino oscillation probabilities within Earth for the calculation of right sign and wrong sign muon events at the detector. For this purpose we need to evaluate new probability amplitudes for neutrino oscillation within two layers of mantle. For example, the probability amplitude for the channel $\nu_{l} \rightarrow \nu_{l^{\prime}}$ with the two layers of mantle considered is expressed as

$$
A_{l l^{\prime}}=\sum_{k, k^{\prime}, k^{\prime \prime}, \alpha, \beta} A_{l k} A_{k k}^{u p}(d) A_{k \alpha} A_{\alpha k^{\prime}} A_{k^{\prime} k^{\prime}}^{l o w}(D) A_{k^{\prime} \beta} A_{\beta k^{\prime \prime}} A_{k^{\prime \prime} k^{\prime \prime}}^{u p}(d) A_{k^{\prime \prime} l^{\prime}}
$$

where $l, l^{\prime}, \alpha, \beta=e, \mu, \tau, s ; k, k^{\prime}, k^{\prime \prime}=1,2,3,4$ and superscripts up (low) correspond to upper and lower mantle with density $\rho_{\text {up }}\left(\rho_{\text {low }}\right)$. Eq. 31 can be explained as follows. The matter effect on neutrinos as they pass through the matter is related to neutrino interaction with matter. The coherent neutrino weak interaction scattering with matter proceeds via weak interaction eigen states or flavour eigen states of neutrinos and depends on the 
particle density (and hence matter density) inside the medium through which the neutrino is propagating (as discussed earlier in Section 2). But it is the mass eigenstates of neutrino in matter (within that medium) which propagate through a distance in that medium. The neutrino in this case, is produced in a particular flavour eigen state in a source and enters the earth matter in the upper mantle with a certain density, through which it will first propagate. The possible mass eigenstates (in matter of the upper mantle) in the initial flavour eigenstate (due to neutrino mixing) is of relevance here. These mass eigenstates initially propagate a distance of $d=1603 \mathrm{~km}$ inside the upper mantle as $\exp (-i E d)(E$ represents the energy eigen value of the neutrino; the propagation Hamiltonian is diagonal in mass basis) till they reach the boundary of upper and lower mantle (Fig. 4). As the lower mantle has a different matter density, the neutrino needs to be converted from its mass eigen state (in upper mantle matter) to possible flavour eigenstates which then enter the lower mantle. But since neutrino will now propagate a distance of $D=4153$ $\mathrm{kms}$ inside the lower mantle with matter density different from that of the upper mantle, these are the mass eigen state(s) in lower mantle matter (and not the flavour eigenstate(s)) that are relevant since these mass eigen states will now propagate as $\exp (-i E D)$ till they reach the boundary of lower mantle and upper mantle (Fig. 4). As they propagate from lower mantle to upper mantle another change of matter density will occur. Following the similar procedure, finally, the neutrino mass eigen states in the upper mantle will reach the detector on earth after traversing a distance of $d=1603 \mathrm{kms}$ (see Fig. 4). But again, since the neutrinos will undergo weak interaction with detector material inside the detector (and a neutrino of a particular flavour will be detected), the relevant flavour eigen state is to be obtained from the mass eigenstates that reach the detector. In Eq. (31), $A_{l k}=\left\langle\nu_{l} \mid \nu_{k}\right\rangle=U_{l k}$ is the element of neutrino mixing matrix before oscillation within matter whereas $A_{k \alpha} \rightarrow U_{k \alpha}\left(\theta_{u p}\right), A_{\alpha k^{\prime}} \rightarrow U_{\alpha k^{\prime}}\left(\theta_{l o w}\right), A_{k^{\prime} \beta} \rightarrow U_{k^{\prime} \beta}\left(\theta_{l o w}\right), A_{\beta k^{\prime \prime}} \rightarrow U_{\beta k^{\prime \prime}}\left(\theta_{u p}\right)$ corresponds to oscillation within matter and $A_{k k}^{u p}=\left\langle\nu_{k} \mid \nu_{k}^{u p}(d)\right\rangle=e^{-i E d}$ with $E$ being a function of $\Delta m_{i j}^{2}, \Delta f_{i j}, V$. Finally the oscillation probability $\nu_{l} \rightarrow \nu_{l^{\prime}}\left(l, l^{\prime}\right.$ are two different neutrino flavours) between two different neutrino flavours can be calculated from the amplitude $P_{l l^{\prime}}=\left|A_{l l^{\prime}}\right|^{2}$.

The computed probabilities for $P_{\bar{e} \bar{\mu}}^{4}$ are plotted in Fig. 5 for three chosen values of $\Delta f_{41}$ namely $\Delta f_{41}=0,10^{-24}$ and $10^{-23}$. As in the case of Figs. 1,2 in Fig. 5 also the upper panel is for Set-1 and the lower panel is for Set-2. Here in the left panel, the computations with $\Delta m_{41}^{2}=1 \times 10^{-3} \mathrm{eV}^{2}$ are shown, while for the right panel $\Delta m_{41}^{2}=3 \times 10^{-3} \mathrm{eV}^{2}$ is adopted. For all the cases, the value of $\Delta f_{31}$ is kept fixed at $\Delta f_{31}=5 \times 10^{-27}$. Similar to what is observed in Figs. 1, 2 here also the VEP effect is more prominent when $\Delta f_{41}=10^{-23}$. But contrary to Figs. 1, 2 in Fig. 5, the VEP effect is prominent when $\Delta m_{41}^{2}=3 \times 10^{-3} \mathrm{eV}^{2}$ is chosen and no appreciable VEP effects are observed when $\Delta m_{41}^{2}=1 \times 10^{-3} \mathrm{eV}^{2}$. Note that, in Figs. 1, 2 no appreciable VEP effects have been observed for $P_{e \mu}^{4}$ when $\Delta m_{41}^{2}=3 \times 10^{-3}$ 
Table 2: The right sign $\mu$ yield and the wrong sign $\mu$ yield in the presence of gravity induced 4-flavour oscillations in matter for Set-1 and for the fixed values of $\Delta f_{31}=$ $5 \times 10^{-27}$. The muon injection energy is fixed at $50 \mathrm{GeV}$. See text for details.

\begin{tabular}{|c|c|c|c|}
\hline$\Delta m_{41}^{2}$ in $\mathrm{eV}^{2}$ & $\Delta f_{41}$ & Right sign $\mu$ & Wrong sign $\mu$ \\
\hline \multirow{3}{*}{$1 \times 10^{-3}$} & 0 & 3115192 & 4182 \\
& $10^{-24}$ & 2973398 & 4176 \\
& $10^{-23}$ & 2662703 & 4543 \\
\hline \multirow{3}{*}{$3 \times 10^{-3}$} & 0 & 3006510 & 4138 \\
& $10^{-24}$ & 2896293 & 3733 \\
& $10^{-23}$ & 2654969 & 5035 \\
\hline
\end{tabular}

Table 3: The right sign $\mu$ yield and the wrong sign $\mu$ yield in the presence of gravity induced 4-flavour oscillations in matter for Set-2 and for the fixed values of $\Delta f_{31}=$ $5 \times 10^{-27}$ with injected muon energy fixed at $50 \mathrm{GeV}$. See text for details.

\begin{tabular}{|c|c|c|c|}
\hline$\Delta m_{41}^{2}$ in $\mathrm{eV}^{2}$ & $\Delta f_{41}$ & Right sign $\mu$ & Wrong sign $\mu$ \\
\hline \multirow{3}{*}{$1 \times 10^{-3}$} & 0 & 3386441 & 4501 \\
& $10^{-24}$ & 2891733 & 4500 \\
& $10^{-23}$ & 1832336 & 6139 \\
\hline \multirow{3}{*}{$3 \times 10^{-3}$} & 0 & 3006875 & 4341 \\
& $10^{-24}$ & 2605874 & 3731 \\
& $10^{-23}$ & 1805902 & 6521 \\
\hline
\end{tabular}




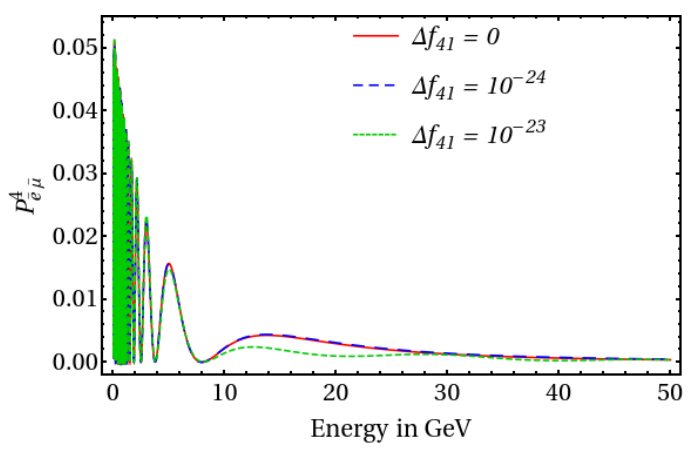

(a) Set-1, $\Delta m_{41}=1 \times 10^{-3} \mathrm{eV}^{2}$

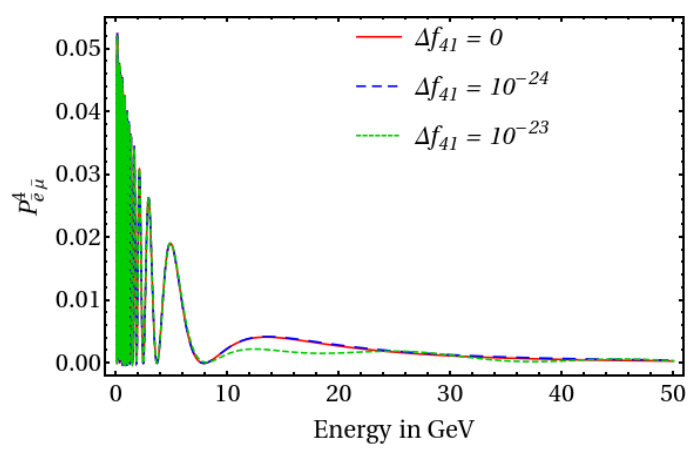

(c) Set-2, $\Delta m_{41}=1 \times 10^{-3} \mathrm{eV}^{2}$

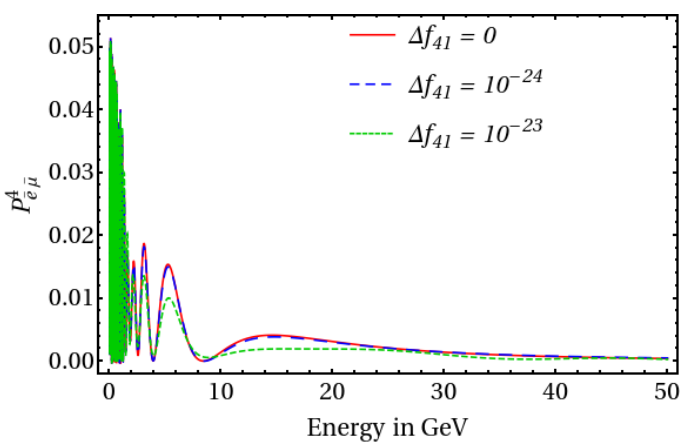

(b) Set-1, $\Delta m_{41}=3 \times 10^{-3} \mathrm{eV}^{2}$

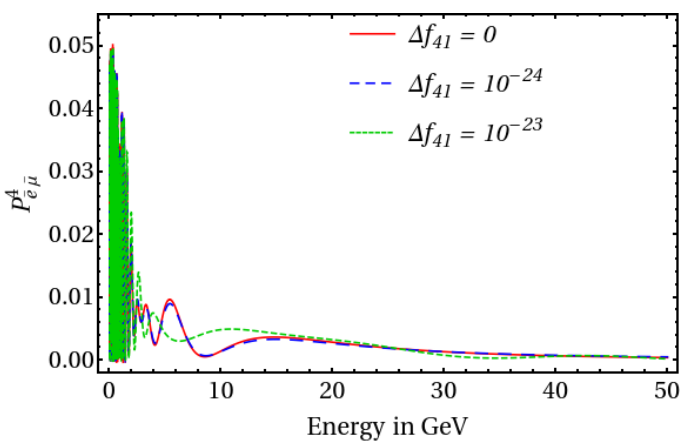

(d) Set-2, $\Delta m_{41}=3 \times 10^{-3} \mathrm{eV}^{2}$

Figure 5: Neutrino oscillation probabilities $\bar{e} \rightarrow \bar{\mu}$ in matter for baseline length $L=$ $7000 \mathrm{~km}$.

$\mathrm{eV}^{2}$ is chosen, but prominent VEP effects are observed when $\Delta m_{41}^{2}=1 \times 10^{-3} \mathrm{eV}^{2}$.

In Table 2, we furnish the estimated right sign and wrong sign muon yields for five year run of a magnetized ICAL detector with the benchmark set of active-sterile mixing angles given in Set-1 of Table 1. We consider the energy of injected muon to be $50 \mathrm{GeV}$ at the muon storage ring directed towards the ICAL detector. The estimated numbers are shown for two values of chosen $\Delta m_{41}^{2}=1 \times 10^{-3} \mathrm{eV}^{2}$ and $\Delta m_{41}^{2}=3 \times 10^{-3} \mathrm{eV}^{2}$. As we have discussed before, keeping $\Delta f_{31}=5 \times 10^{-27}$ fixed, we compute the right sign and wrong sign muon yields at the ICAL detector considered here for different $\Delta f_{41}$ values discussed in previous section. From Table 2, we observe that for both the values of $\Delta m_{41}^{2}=1(3) \times 10^{-3}$ $\mathrm{eV}^{2}$, increase in $\Delta f_{41}$ results in depletion of the right sign muon yields while wrong sign muon yields do not suffer any significant change. We also compute the right and wrong sign muon yields for a set of different benchmark points for active-sterile mixing given in Set 2 of Table 1 keeping the other parameters same as those used in Table 2. The results are furnished in Table 3. Similar trend as in Table 2 is also observed for the computed right and wrong sign muon yield values given in Table 3 . From these calculations it appears that long baseline neutrino experiment can be very effective and important for not only to 
Table 4: Comparison of the right sign and the wrong sign $\mu$ yields for the 4-flavour case compared to the same for 3-flavour case for two sets of active-sterile neutrino mixing angles and for two different values of $\Delta m_{41}^{2}$. We consider $\Delta f_{41}=0$ for this comparison and muon injection energy is kept fixed at $50 \mathrm{GeV}$. See text for details.

\begin{tabular}{|c|c|c|c|c|c|c|c|}
\hline $\begin{array}{c}\Delta m_{41}^{2} \\
\text { in } \mathrm{eV}^{2}\end{array}$ & $\theta_{14}$ & $\theta_{24}$ & $\theta_{34}$ & $\begin{array}{c}\text { Right } \\
\text { sign } \mu \\
\text { in } \\
\text { 4-flavour }\end{array}$ & $\begin{array}{c}\text { Wrong } \\
\text { sign } \mu \\
\text { in } \\
\text { 4-flavour }\end{array}$ & $\begin{array}{c}\text { Right } \\
\text { sign } \mu \\
\text { in } \\
\text { 3-flavour }\end{array}$ & $\begin{array}{c}\text { Wrong } \\
\text { sign } \mu \\
\text { in } \\
\text { 3-flavour }\end{array}$ \\
\hline \multirow{2}{*}{$1 \times 10^{-3}$} & $3.6^{\circ}$ & $4.0^{\circ}$ & $18.48^{\circ}$ & 3115192 & 4182 & 2250268 & 463 \\
& $2.5^{\circ}$ & $10.0^{\circ}$ & $30.0^{\circ}$ & 3386441 & 4501 & 2250268 & 463 \\
\hline \multirow{2}{*}{$3 \times 10^{-3}$} & $3.6^{\circ}$ & $4.0^{\circ}$ & $18.48^{\circ}$ & 3006510 & 4138 & 2250268 & 463 \\
& $2.5^{\circ}$ & $10.0^{\circ}$ & $30.0^{\circ}$ & 3006875 & 4341 & 2250268 & 463 \\
\hline
\end{tabular}

address, in addition to other neutrino physics issues, the four neutrino flavour scenario but also to probe a possible tiny violation of equivalence principle, if any.

In order to demonstrate, how both the right sign and wrong sign muon yields for 4flavour scenario differ from those when only three active flavours are considered with and without VEP effects, we compare in Table 4 the yields for the two cases by computing the right sign and wrong sign muon yields when three flavour mixing parameters are same for both the scenarios. The other parameters of active-sterile mixing are adopted as given in Sets I and II of Table I (and also shown in Table 4). The value of $\Delta f_{31}=5 \times 10^{-27}$, as in Tables 2 and 3 and fixed from the bounds on $\Delta f_{31}$ given in Ref. [47] and $\Delta f_{41}=0$. All computations are for five year run of the chosen 50kTon ICAL detector assumed to be placed at a distance of $7359 \mathrm{~km}$ from a neutrino factory with muon injection energy of 50 $\mathrm{GeV}$.

From Table 4, it is clear that for both the cases of right sign and wrong sign muon yields are enhanced for 4 -flavour ( 3 active +1 sterlile) scenario when compared with 3 flavour oscillation. The difference is more striking for the case of wrong sign muon yield. For example, when $\Delta m_{41}^{2}=3 \times 10^{-3}$ is chosen, the wrong sign muon yield for 4-flavour case is 20 times larger in magnitude than the same for 3 -flavour case. If the active-sterile VEP oscillation is made non-zero $\left(\Delta f_{41} \neq 0\right)$, the 4 -flavour results will be modified as seen from Tables 2 and 3 but the similar trend in difference of wrong sign muon yields are maintained. However, for increasing $\Delta f_{41}$, right sign muon yield reduces considerably and can become even smaller than the 3 -flavour case as observed in Table 3 for $\Delta f_{41}=10^{-23}$. From the estimated results of muon yields obtained in Table 2-3 at the chosen ICAL detector, it is now evident that the presence of gravity induced four neutrino oscillations in matter will 
significantly affect the right sign and wrong sign muon yields for non zero $\Delta f_{41}$. Therefore, long baseline neutrino experiment can be a viable probe to investigate the violation of equivalence principle appearing in four flavour scenario.

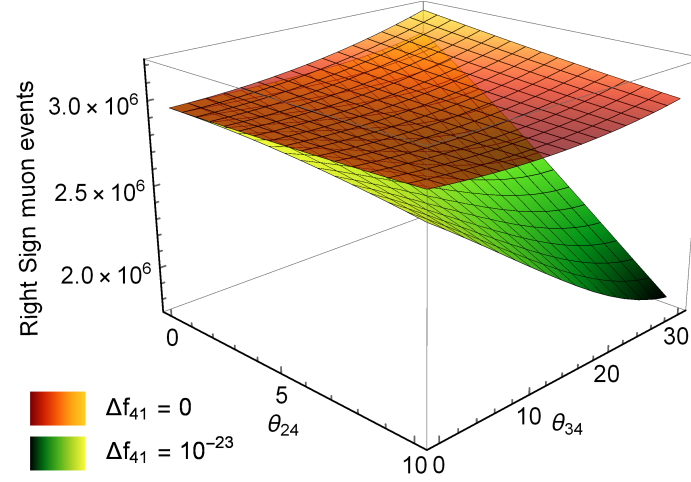

(a) Variation of right sign $\mu$ yield

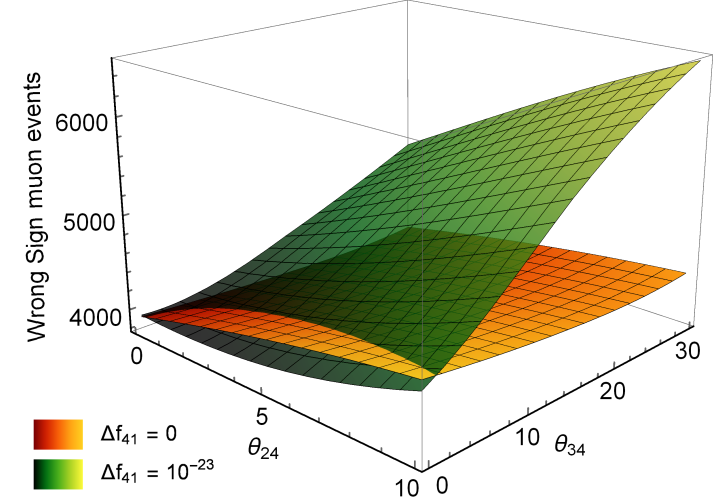

(b) Variation of wrong sign $\mu$ yield

Figure 6: Right sign and wrong sign muon yield variations with $\theta_{24}$ and $\theta_{34}$ for fixed $\theta_{14}=2.5^{0}, \Delta f_{31}=5 \times 10^{-27}$ and two chosen values of $\Delta f_{41}=0,10^{-23}$. See text for details.

Finally the 3D plots in Fig. 6, we show the simultaneous variations of each of the right sign and wrong sign muon yields with $\theta_{24}$ (ranging from $0^{0} \leq \theta_{24} \leq 10^{0}$ ) and $\theta_{34}$ (ranging from $0^{0} \leq \theta_{34} \leq 30^{0}$ ) for fixed values of $\theta_{14}=2.5^{0}$ and $\Delta f_{31}=5 \times 10^{-27}$. The chosen ranges of $\theta_{24}$ and $\theta_{34}$ are within the allowed regions given by 4 -flavour analyses of oscillation experiment data. We compute the muon yields with muon injection energy 50 $\mathrm{GeV}$ in proposed ICAL detector with baseline length $7359 \mathrm{~km}$ for five year run and compare the muon yield values for $\Delta m_{41}^{2}=10^{-3} \mathrm{eV}^{2}, \Delta f_{41}=0$ with $\Delta f_{41}=10^{-23}$. In Fig. 6a, we notice that for $\Delta f_{41}=0$, right sign muon yield does not change significantly with the variations of $\theta_{24}$ and $\theta_{34}$. However, with $\Delta f_{41}=10^{-23}$, one observes considerable depletion in right sign muon yield as $\theta_{34}$ increases while the changes are negligible with the variations of $\theta_{24}$. This result is also in agreement with the muon yield events reported earlier in Table 2-4, where a reduction has been observed in right sign muon yield for increase in $\Delta f_{41}$. A similar plot with the same set of parameters for wrong sign muon yield events at ICAL detector with $50 \mathrm{GeV}$ muon injection energy (and five year run) is shown in Fig. 6b. Comparing the values of wrong sign muon yields for $\Delta f_{41}=0$ with $\Delta f_{41}=10^{-23}$, we observe very mild variation in wrong sign muon yields as the values of $\theta_{24}, \theta_{34}$ are changed. The nature of this $3 \mathrm{D}$ plot is consistent with the results shown in Tables 2-4 for wrong sign muons. Thus, this may be concluded that in case of 4-flavour oscillations the VEP, if exists in nature can influence considerable effect on muon yields in a long baseline neutrino experiment. 


\section{Summary and Discussions}

If the equivalence principle is indeed violated in nature, this will induce different gravitational couplings for different types of neutrinos. Here in this work, we study how such a probability affects the neutrino oscillations in matter and the possibility that even a very small violation of equivalence principle can be probed by a long baseline neutrino experiment. To this end we consider a four neutrino $(3+1)$ framework, where one extra sterile neutrino is assumed to exist in addition to the three active neutrinos. In a possible scenario that the equivalence principle is violated in nature, the three active neutrinos as well as the sterile neutrino couple differently with gravity which result in a gravity induced oscillations of neutrinos in addition to mass flavour oscillation of neutrinos. In addition, one must also take into account the mater effects if neutrinos propagate through medium. In this work, we derive the effective Hamiltonian for four neutrino oscillations in presence of gravity induced effects along with usual mass-flavour oscillations with matter effects. we then study the effects of VEP in a long baseline neutrino oscillation experiment, by estimating the detector yields at the far detector.

We derive the new oscillation probabilities for neutrino oscillations within matter assuming the mixing angles between mass and flavour eigenstates to be identical with those between gravity and flavour eigenstates. The above choice allows us to study the effects of new parameters $\Delta f_{31}, \Delta f_{41}$ which are responsible for gravity induced neutrino oscillations and can be attributed to the signature and measure of violation of equivalence principle. However, IceCube data of atmospheric neutrino puts stringent constraint on $\Delta f_{31}$. Using the bounds on neutrino mixing angles from different experiments and $\Delta f_{31}$, we study the behaviour of four neutrino oscillation probabilities considering a representative long baseline of $7000 \mathrm{~km}$ for different values of $\Delta f_{41}$ while the values of $\Delta m_{41}^{2}$ and $\Delta f_{31}$ are kept fixed. For demonstrative purpose, we consider two benchmark sets of active-sterile neutrino mixing angles $\theta_{14}, \theta_{24}, \theta_{34}$. We observe that significant deviations in oscillation probabilities $P_{\nu_{\alpha} \rightarrow \nu_{\beta}}$ occur with the changes in $\Delta f_{41}$ indicating that even a very weak violation of the equivalence principle will affect the oscillation probabilities over a chosen representative baseline of $7000 \mathrm{~km}$.

With oscillation probabilities that we derive in this work for 3 active and 1 sterile neutrino formalism, we make an estimate of the signatures of violation of equivalence principle at the end detector of an assumed LBL neutrino experiment where the neutrinos are produced in a neutrino factory and are detected at a far detector of magnetized iron calorimeter (ICAL) with a baseline length of around $7359 \mathrm{~km}$. The magnetized ICAL detector can efficiently measure the number of $\mu^{-}$and $\mu^{+}$produced upon charged current interaction of muon neutrinos and muon anti-neutrinos at the detector. Flux of muon neutrinos $\left(\nu_{\mu}\right)$ will suffer gravity induced and mass induced oscillations in matter while propagating to the far 
ICAL detector from the neutrino factory and thus it will be depleted. Hence this oscillation channel is referred to as disappearance channel. Similarly detection of $\mu^{+}$at magnetized ICAL indicates the appearance channel due to neutrino oscillation $\bar{\nu}_{e} \rightarrow \bar{\nu}_{\mu}$. Our study reveals that, since $\Delta f_{31} \simeq 10^{-27}$, VEP effect is negligible in case of three flavour neutrino oscillations and also negligible in case of four flavour oscillations when $\Delta f_{41}=0$ is taken into account. From the calculation of various oscillation probabilities, we demonstrate that effect of VEP becomes prominent with four flavour oscillations for $\Delta f_{41}=10^{-23}$. This is further justified by the calculation of muon yields $\left(\mu^{\mp}\right)$ in a proposed long baseline neutrino experiment with baseline length $7359 \mathrm{~km}$. The results show significant changes in muon yields arising due to VEP parameter $\Delta f_{41}$ responsible for gravity induced neutrino oscillations. In case of four flavour oscillations, we conclude that the effect of VEP is distinguished significantly for $\Delta f_{41}=10^{-23}$. In fact, effect of gravity induced oscillations of neutrinos in four flavour scenario is determined entirely by the parameter $\Delta f_{41}$. Therefore, long baseline neutrino experiment can be used to probe even a very small violation of equivalence principle, if exists in nature. However, non observation of any such deviations in predicted muon yield will certainly rule out the possibility of the effect of VEP in neutrino oscillations. It is to be noted that one can also perform a detailed study of VEP effect where sterile neutrino is considered and put limit on $\Delta f_{41}$, similar to the work done in Ref. [47] to constrain $\Delta f_{31}$. However, such an analysis is beyond the scope of present work and can be pursued in a future work.

Acknowledgements : Authors acknowledge A. Bandyopadhyay for useful discussions. One of the authors (M.P.) thanks the DST-INSPIRE fellowship (DST/INSPIRE Fellowship/2016/IF160004) grant by Department of Science and Technology (DST), Govt. of India. One of the authors (A.H.) acknowledges the support received from St. Xavier's College, Kolkata Central Research Facility and also thanks the University Grant Commission (UGC) of the Government of India, for providing financial support, in the form of UGCCSIR NET-JRF. ADB acknowledges the support and hospitality of SINP for the completion of the work. Work of ADB is supported in part by the National Science Foundation of China $(11422545,11947235)$.

\section{References}

[1] S.M. Bilenky and B. Pontecorvo, Phys. Rep. 41, 225 (1978).

[2] T.A. Mueller et al., Phys. Rev. C 83, 054615 (2011).

[3] P. Huber, Phys. Rev. C 84, 024617 (2011).

[4] G. Mention et al., Phys. Rev. D 83, 073006 (2011). 
[5] M. Gasperini, Phys. Rev. D 38, 2635 (1988).

[6] M. Gasperini, Phys. Rev. D 39, 12 (1989).

[7] M. Gasperini, Phys. Rev. D 38, 9 (1988).

[8] L. Landau and E. Lifchitz, Theorie du Champ (MIR, Moscow, 1966), Sec. 89.

[9] A.D. Banik and D. Majumdar, Mod. Phys. Lett. A 30, 1550001 (2015).

[10] S. Ahmed et al. [ICAL Collaboration], Pramana 88, no. 5, 79 (2017); http://www.ino.tifr.res.in/ino/

[11] B. Pontecorvo, Sov. Phys. JTEP 6, 429 (1958).

[12] B. Pontecorvo, Sov. Phys. JTEP 7, 172 (1958).

[13] Z. Maki, M. Nakagawa, S. Sakata, Prog. Theo. Phys., 28, (1962).

[14] S.K. Kang et al. Hinsawi Publishing Corporation 2013, 138109 (2013).

[15] M. Pandey, JHEP 01, 066, (2019).

[16] C. Giunti, C.W. Kim and M. Monteno, Nucl. Phys. B 521, 3 (1998).

[17] E.Kh. Akhmedov, arXiv:hep-ph/0001264.

[18] L. Wolfenstein, Phys. Rev. D 17, 2369 (1978).

[19] S.P. Mikheyev, A.Yu. Smirnov, Sov. J. Nucl. Phys. 42, 913 (1985).

[20] M. Tanabashi et al. [Particle Data Group], Phys. Rev. D 98, no. 3, 030001 (2018).

[21] P. Adamson et al. (MINOS Collaboration), Nucl. Instrum. Meth. A 806, 279 (2016).

[22] P. Adamson et al. ( MINOS Collaboration), Phys. Rev. Lett. 117, 151803 (2016).

[23] P. Adamson et al. [MINOS+ Collaboration], Phys. Rev. Lett. 122, no. 9, 091803 (2019).

[24] P. Adamson et al. (MINOS Collaboration), Phys. Rev. Lett. 110, 171801 (2013).

[25] P. Adamson et al. (MINOS Collaboration), Phys. Rev. Lett. 107, 011802 (2011).

[26] D.G. Michael et al. (MINOS Collaboration), Phys. Rev. Lett. 97, 191801 (2006).

[27] P. Adamson et al. (MINOS Collaboration), Phys. Rev. Lett. 101, 221804 (2008).

[28] P. Adamson et al. (MINOS Collaboration), Phys. Rev. Lett. 112, 191801 (2014). 
[29] P. Adamson et al. (MINOS Collaboration), Phys. Rev. D 81, 052004 (2010).

[30] D.G. Michael et al. (MINOS Collaboration), Nucl. Instrum. Methods Phys. Res., Sect. A 596, 190 (2008).

[31] P. Adamson et al. (MINOS Collaboration), Phys. Rev. D 77, 072002 (2008).

[32] S. De Rijck [MINOS and MINOS+ Collaborations], J. Phys. Conf. Ser. 873, no. 1, 012032 (2017).

[33] P. Adamson et al. [Daya Bay and MINOS Collaborations], Phys. Rev. Lett. 117, no. 15, 151801 (2016).

[34] F.P. An et al. (DAYA-BAY Collaboration), Phys. Rev. Lett. 108, 171803 (2012).

[35] F.P. An et al. (DAYA-BAY Collaboration), following Letter, Phys. Rev. Lett. 117, $171802(2016)$.

[36] F.P. An et al. (DAYA-BAY Collaboration), Nucl. Instrum. Methods Phys. Res., Sect. A 811, 133 (2016).

[37] F.P. An et al. (DAYA-BAY Collaboration), Phys. Rev. Lett. 112, 061801 (2014).

[38] F.P. An et al. (DAYA-BAY Collaboration), Phys. Rev. Lett. 116, 061801 (2016).

[39] F.P. An et al. (DAYA-BAY Collaboration), Phys. Rev. Lett. 115, 111802 (2015).

[40] F.P. An et al. (DAYA-BAY Collaboration), Phys. Rev. Lett. 113, 141802 (2014).

[41] B. Achkar et al. (BUGEY Collaboratrion), Nucl. Phys. B 434, 503 (1995).

[42] K. Abe et al. [T2K Collaboration], Phys. Rev. D 99, no. 7, 071103 (2019).

[43] M. G. Aartsen et al. [IceCube Collaboration], Phys. Rev. D 95, no. 11, 112002 (2017).

[44] M. Adams, F. Bezrukov, J. Elvin-Poole, J. J. Evans, P. Guzowski, B. Ó. Fearraigh and S. Söldner-Rembold, arXiv:2002.07762 [hep-ph].

[45] D. Majumdar, A. Ghosal, Phys. Rev. D 75, 113004 (2007).

[46] M. Pandey, D. Majumdar and A.D. Banik, Phys. Rev. D 97, 103015 (2018).

[47] A. Esmaili, D. R. Gratieri, M. M. Guzzo, P. C. de Holanda, O. L. G. Peres and G. A. Valdiviesso, Phys. Rev. D 89, no.11, 113003 (2014).

[48] S. Geer, Phys. Rev. D 57, 6989 (1998). 
[49] A. Donini, D. Meloni and P. Migliozzi, Nucl. Phys. B 646, 321 (2002).

[50] A. M. Dziewonski and D. L. Anderson, Phys. Earth Planet. Interiors 25, 297-356 (1981). 